\title{
Comparison of improved class of priors for the analysis of the Burr type VII model under doubly censored samples
}

\author{
Navid Feroze*1,2 (iD, Muhammad Aslam ${ }^{1}$ \\ ${ }^{1}$ Department of Mathematics and Statistics, Riphah International University, Islamabad, Pakistan \\ ${ }^{2}$ Department of Statistics, The University of Azad Jammu and Kashmir, Muzaffarabad, Pakistan
}

\begin{abstract}
In recent years, the finite mixture lifetime models have frequently been used in chemical, physical, social science, biological and other fields due to their methodological development and practical applications. The Bayesian analysis of the mixture models has also developed a significant interest among the statisticians especially in the last decade. However, the most of these contributions are limited to the Bayes estimation for the parameters of lifetime models under singly type I censoring. This paper discusses the Bayesian estimation for the two-component mixture of the lifetime models under doubly censored samples with a particular case for the Burr type VII model. A class of improved priors has been proposed for the posterior estimation. The likelihood function, for doubly censored samples using two-component mixture of life time distributions, has been introduced. The hazard rate function for the mixture model has been compared for different parametric values. The performance of various estimators has been compared under a simulation study along with a real life example.
\end{abstract}

Mathematics Subject Classification (2020). 62F15, 62N01

Keywords. Mixture models, posterior distributions, loss functions, credible intervals, posterior predictive intervals

\section{Introduction}

Burr [5] introduced a family of Burr distribution including twelve life time distributions. These distributions can be used to model almost all kinds of failure time data. Among these distributions, the Burr type VII distribution has rarely been considered by the analysts, maybe due to its complex density function. This distribution can also be used for analysis of the life time data. Recently, Feroze and Aslam [10] discussed some properties of the distribution under a Bayesian framework using left, singly type II and doubly type II censored samples. The distribution still requires much attention to explore its applications in different real life situations. To deal with characteristics of such unfamiliar distributions is always of great interest for the researchers. The insight we can get from them can be beneficial to the professionals looking to use those distributions as models.

\footnotetext{
*Corresponding Author.

Email addresses: navidferoz@gmail.com (N. Feroze), maslamdqu@gmail.com (M. Aslam)

Received: 16.06.2015; Accepted: 23.01.2017
} 
Among the other members from the family of Burr distributions; the Burr type X and XII have received the sizable attention of the researchers. For further details regarding these contributions, the readers may refer to: [11, 12, 28, 33, 40-44, 46, 49-51].

The finite mixture models are typically used to model data where each observation is assumed to have come from more than one groups (sub-populations), each group being suitably modeled by a density from some parametric family. The density representing each group is referred to as a component of the mixture. These components are mixed according to their proportions (relative frequencies) in the population. These mixture models provide a convenient and flexible family of distributions for estimating or approximating distributions which are not well modeled by any standard family, such as kernel density estimation. In recent years, the finite mixture lifetime models have frequently been used in chemical, physical, social science, biological and other fields due to their methodological development and practical applications. These mixture models can analyze different datasets when a population is categorized in number of sub-populations based on different causes of failures. For example, a population of lifetimes of certain electrical elements can be classified into number of sub-populations due to different causes of failures. Several papers have been appeared on classical analysis of the mixture of life time distributions under complete and censored data, these include: [1, 2, 8, 9, 19,23, 24, 26, 27, 30-32, 45, 47].

The analysis of mixture models under Bayesian framework has developed a significant interest among the statisticians. The authors dealing with Bayesian analysis of mixture models include: [25,36-39]. But these contributions are limited to the Bayes point estimation of the parameters under singly type I censored data. In singly type I censoring there is one test termination point; however an analyst may be interested to have two test termination times in situations where the equipment age is unknown at the start of a period of equipment monitoring and the tracking of the equipment is stopped after a predetermined observation interval. In such situation the use of doubly censored samples can effectively be used. Doubly censoring is the device used to describe the methodologies for analyzing duration times between two events. In this censoring, it is necessary to define two time points: the origin time corresponding to the time at which an original event occurs and the failure time corresponding to the time at which the final event occurs. For example, in the context of HIV/AIDS studies starting time may be taken as the infection time of a patient, the termination time can be the time of the AIDS diagnosis of the patients. The patients which at the end of the study have not developed the disease may be considered as right censored. The elapsed time between these two points is the AIDS latency time. For more illustrations on doubly type-II censoring, the reader may refer to: $[12,15,29,34,35,48]$.

In this paper, the two-component mixture of the Burr type VII has been considered under doubly type II censored samples. An extended version of the likelihood function under doubly type II censored samples has been introduced for two-component mixture of lifetime distributions. A class of improved informative priors has been proposed for the posterior analysis. The Bayes point and interval estimators along with posterior predictive intervals have been derived and evaluated. The proposed method can be extended for more than two component mixtures.

The organization of the paper is as follows: Section 2 contains of the model and the introduction of likelihood function for two-component mixture of Burr type VII distribution under doubly censored samples. The improved priors have been introduced and posterior distributions have been derived in Section 3. Bayes point estimation has been discussed in Section 4. The algorithm for prior elicitation has been given in Section 5 . The results of the simulation study have been presented in Section 6. A real life example has been used to discuss the applicability of the results in Section 7. The hazard rate function for the mixture model has been discussed in Section 8. Finally, the conclusions regarding the study have been presented in Section 9 . 


\section{The model and likelihood function}

The probability density function (pdf) of the Burr type VII distribution is

$$
\begin{aligned}
f_{j}\left(x_{j i}\right) & =\theta_{j} 2^{-\theta_{j}} \operatorname{sech}^{2}\left(x_{j i}\right)\left\{1+\tanh \left(x_{j i}\right)\right\}^{\theta_{j}-1}, \\
& -\infty<x_{j i}<\infty, \theta_{j}>0, j=1,2 i=1,2, \ldots, n .
\end{aligned}
$$

The cumulative distribution function $(\mathrm{CDF})$ of the distribution is

$$
\begin{aligned}
F_{j}\left(x_{j i}\right) & =2^{-\theta_{j}}\left\{1+\tanh \left(x_{j i}\right)\right\}^{\theta_{j}}, \\
& -\infty<x_{j i}<\infty, \theta_{j}>0, j=1,2 i=1,2, \ldots, n .
\end{aligned}
$$

A density function for mixture of two components densities with mixing weights $(\pi, 1-\pi)$ is

$$
f(x)=\pi f_{1}(x)+(1-\pi) f_{2}(x), 0<\pi<1 .
$$

The cumulative distribution function for the mixture model is

$$
F(x)=\pi F_{1}(x)+(1-\pi) F_{2}(x) .
$$

Consider a random sample of size $n$ from Burr type VII distribution and let $x_{r}, x_{r}+$ $1, \ldots, x_{s}$ be the ordered observations that can only be observed. The remaining $r-1$ smallest observations and the $n-s$ largest observations have been assumed to be censored. Now based on causes of failure, the failed items are assumed to come either from subpopulation 1 or from sub-population 2 ; so that the $x_{1 r_{1}}, \ldots, x_{1 s_{1}}$ and $x_{2 r_{2}}, \ldots, x_{2 s_{2}}$ failed items come from first and second sub-populations respectively. The rest of the observations which are less than $x_{r}$ and greater than $x_{s}$ have been assumed to be censored from each component where $x_{s}=\max \left(x_{1, s_{1}}, x_{2, s_{2}}\right)$ and $x_{r}=\min \left(x_{1, r_{1}}, x_{2, r_{2}}\right)$. Therefore, $m_{1}=$ $s_{1}-r_{1}+1$ and $m_{2}=s_{2}-r_{2}+1$ number of failed items can be observed from first and second sub-population respectively. The remaining $m=s-r+1$ items are assumed to be censored observations where $r=r_{1}+r_{2}, s=s_{1}+s_{2}$ and $m=m_{1}+m_{2}$. Then the likelihood function for the type II doubly censored sample $\mathrm{x}=\left\{\left(x_{1 r_{1}}, \ldots, x_{1 s_{1}}\right),\left(x_{2 r_{2}}, \ldots, x_{2 s_{2}}\right)\right\}$, assuming the causes of the failure of the left censored items are identified, can be written as

$$
\begin{aligned}
L\left(\theta_{1}, \theta_{2}, \pi \mid \mathrm{x}\right) \propto & \left\{F\left(x_{1, r_{1}}\right)\right\}^{r_{1}-1}\left\{F\left(x_{2, r_{2}}\right)\right\}^{r_{2}-1}\left\{1-F\left(x_{s}\right)\right\}^{n-s} \\
& \times\left\{\prod_{i=r_{1}}^{s_{1}} f\left(x_{1, i}\right)\right\}\left\{\prod_{i=r_{2}}^{s_{2}} f\left(x_{2, i}\right)\right\} .
\end{aligned}
$$

Putting vales in Equation (2.5) and simplifying, we get

$$
\begin{aligned}
L\left(\theta_{1}, \theta_{2}, \pi \mid \mathrm{x}\right) \propto \sum_{k_{1}=0}^{r_{1}-1} \sum_{k_{2}=0}^{r_{2}-1} \sum_{k_{3}=0}^{n-s} \sum_{k_{4}=0}^{k_{3}}(-1)^{k_{3}}\left(\begin{array}{c}
r_{1}-1 \\
k_{1}
\end{array}\right)\left(\begin{array}{c}
r_{2}-1 \\
k_{2}
\end{array}\right)\left(\begin{array}{c}
n-s \\
k_{3}
\end{array}\right)\left(\begin{array}{c}
k_{3} \\
k_{4}
\end{array}\right) \pi^{k_{1}+k_{3}+k_{4}} \\
\times(1-\pi)^{r_{1}+r_{2}+k_{3}-k_{1}-k_{2}-k_{4}-2} \theta_{1}^{m_{1}} e^{-\theta_{1} \Omega\left(x_{1 i}\right)} \theta_{2}^{m_{2}} e^{-\theta_{2} \Omega\left(x_{2 i}\right)}
\end{aligned}
$$

where

$\Omega\left(x_{1 i}\right)=-\sum_{i=r_{1}}^{s_{1}} \ln \left\{\frac{1+\tanh \left(x_{1 i}\right)}{2}\right\}-k_{1} \ln \left\{\frac{1+\tanh \left(x_{1, r_{1}}\right)}{2}\right\}-k_{2} \ln \left\{\frac{1+\tanh \left(x_{2, r_{2}}\right)}{2}\right\}-k_{4} \ln \left\{\frac{1+\tanh \left(x_{s}\right)}{2}\right\}$ and

$\Omega\left(x_{2 i}\right)=-\sum_{i=r_{2}}^{s_{2}} \ln \left\{\frac{1+\tanh \left(x_{2 i}\right)}{2}\right\}-\left(r_{1}-k_{1}-1\right) \ln \left\{\frac{1+\tanh \left(x_{1, r_{1}}\right)}{2}\right\}-\left(r_{2}-k_{2}-1\right) \ln \left\{\frac{1+\tanh \left(x_{2, r_{2}}\right)}{2}\right\}-\left(k_{3}-k_{4}\right) \ln \left\{\frac{1+\tanh \left(x_{s}\right)}{2}\right\}$.

This can further be written as

$$
\begin{gathered}
L\left(\theta_{1}, \theta_{2}, \pi \mid \mathrm{x}\right) \propto \sum_{k_{1}=0}^{r_{1}-1} \sum_{k_{2}=0}^{r_{2}-1} \sum_{k_{3}=0}^{n-s} \sum_{k_{4}=0}^{k_{3}}\left(\begin{array}{c}
r_{1}-1 \\
k_{1}
\end{array}\right)\left(\begin{array}{c}
r_{2}-1 \\
k_{2}
\end{array}\right)\left(\begin{array}{c}
n-s \\
k_{3}
\end{array}\right)\left(\begin{array}{c}
k_{3} \\
k_{4}
\end{array}\right) \prod_{w=1}^{2}(-1)^{k_{3}} \\
\times \pi^{k_{1}+k_{3}+k_{4}}(1-\pi)^{r_{1}+r_{2}+k_{3}-k_{1}-k_{2}-k_{4}-2} \theta_{w}^{m_{w}} e^{-\theta_{w} \Omega\left(x_{w i}\right)} .
\end{gathered}
$$




\section{Prior and posterior distributions}

There are several rules for defining a non-informative prior, such as the principle of indifference and the Jeffreys rule. The principle of indifference is undoubtedly the simplest and oldest rule. Using the principle of indifference, Laplace [20] has proposed one of the most widely used non-informative prior, namely uniform prior. The expressions of uniform prior for the parameters of the mixture model can be given as: $g\left(\theta_{1}, \theta_{2}, \pi\right) \propto 1$. But it seems to be inadequate to the specific situation. As prior is often used when the parameter $\theta$ is defined on $(-\infty, \infty)$. If the parametric range is restricted to be positive, then a noninformative prior satisfying the Jeffreys rule is often considered as: $g(\theta) \propto \theta^{-1}$. Whereas, if $\theta$ is defined in $(0,1)$, the non-informative prior is usually defined as: $g(\theta) \propto \theta^{-\frac{1}{2}}(1-\theta)^{-\frac{1}{2}}$. Whenever a specific rule is not defined and the resulting non-informative prior cannot explicitly be derived, the following vague joint prior can be used for the parameters of the mixed Burr type VII distribution:

$$
g\left(\theta_{1}, \theta_{2}, \pi\right) \propto \theta_{1}^{-1} \theta_{2}^{-1} \pi^{-1 / 2}(1-\pi)^{-1 / 2} ; \theta_{1}, \theta_{2}>0
$$

The current literature regarding Bayesian analysis of the mixture models mostly includes the assumption of non-informative uniform prior for the mixing parameter along with informative priors for the parameters representing the different components of the model (see $[25,36-39]$ ). We have proposed the informative beta prior for the mixing parameter, which has been combined with other priors to produce the improved informative priors for the posterior analysis. The description of these priors has been given in the following.

Let $\theta_{1} \sim \operatorname{Gamma}\left(a_{1}, b_{1}\right)$ and $\theta_{2} \sim \operatorname{Gamma}\left(a_{2}, b_{2}\right)$ are the gamma priors for each parameter and $\pi \sim \operatorname{Beta}\left(a_{3}, b_{3}\right)$ is the beta prior for mixing parameter $\pi$. Under the assumption of independence, these priors have been combined to produce a joint improved gamma prior for parameter as

$$
\begin{aligned}
& g\left(\theta_{1}, \theta_{2}, \pi\right) \propto \theta_{1}^{a_{1}-2} \theta_{2}^{a_{2}-2} e^{-\left(\theta_{1} b_{1}+\theta_{2} b_{2}\right)} \pi^{a_{3}-1}(1-\pi)^{b_{3}-1}, \\
& \theta_{1}, \theta_{2}>0,0<\pi<1, a_{1}, a_{2}, a_{3}, b_{1}, b_{2}, b_{3}>0
\end{aligned}
$$

where $a_{1}, a_{2}, a_{3}, b_{1}, b_{2}$ and $b_{3}$ are hyper-parameters. Another informative prior is the Jeffreys gamma prior suggested by [18]. Let $\theta_{1} \sim \operatorname{JeffreysGamma}\left(a_{1}, b_{1}\right)$ and $\theta_{2} \sim$ JeffreysGamma $\left(a_{2}, b_{2}\right)$ are the Jeffreys gamma priors for each parameter and $\pi \sim \operatorname{Beta}\left(a_{3}, b_{3}\right)$ is the beta prior for mixing parameter $\pi$. Under the assumption of independence, these priors have been combined to produce a joint improved Jeffreys gamma prior for parameter as

$$
\begin{array}{r}
g\left(\theta_{1}, \theta_{2}, \pi\right) \propto \theta_{1}^{a_{1}-2} \theta_{2}^{a_{2}-2} e^{-\left(\theta_{1} b_{1}+\theta_{2} b_{2}\right)} \pi^{a_{3}-1}(1-\pi)^{b_{3}-1} \\
\theta_{1}, \theta_{2}>0,0<\pi<1, a_{1}, a_{2}, a_{3}, b_{1}, b_{2}, b_{3}>0
\end{array}
$$

where $a_{1}, a_{2}, a_{3}, b_{1}, b_{2}$ and $b_{3}$ are hyper-parameters. Suppose $\theta_{1} \sim \operatorname{InverseLevy~}\left(b_{4}\right)$, $\theta_{2} \sim$ InverseLevy $\left(b_{5}\right)$ and $\pi \sim \operatorname{Beta}\left(a_{3}, b_{3}\right)$ then these marginal priors can be united to introduce a joint improved inverse levy prior as

$$
\begin{gathered}
g\left(\theta_{1}, \theta_{2}, \pi\right) \propto\left(\theta_{1} \theta_{2}\right)^{-0.5} e^{-0.5\left(b_{4} \theta_{1}+b_{5} \theta_{2}\right)} \pi^{a_{3}-1}(1-\pi)^{b_{3}-1}, \\
\theta_{1}, \theta_{2}>0,0<\pi<1, a_{3}, b_{3}, b_{4}, b_{5}>0
\end{gathered}
$$

where $a_{3}, b_{3}, b_{4}$ and $b_{5}$ are hyper-parameters. Further, suppose $\theta_{1} \sim$ Exponential $\left(d_{1}\right)$, $\theta_{2} \sim$ Exponential $\left(d_{2}\right)$ and $\pi \sim \operatorname{Beta}\left(a_{3}, b_{3}\right)$ then these marginal priors can be combined to introduce a joint improved exponential prior as

$$
\begin{aligned}
& g\left(\theta_{1}, \theta_{2}, \pi\right) \propto e^{-\left(d_{1} \theta_{1}+d_{2} \theta_{2}\right)} \pi^{a_{3}-1}(1-\pi)^{b_{3}-1}, \\
& \theta_{1}, \theta_{2}>0,0<\pi<1, a_{3}, d_{1}, d_{2}>0
\end{aligned}
$$


where $a_{3}, d_{1}$ and $d_{2}$ are hyper-parameters. Finally, consider $\theta_{1} \sim$ ChiSquare $\left(d_{3}\right)$ and $\pi \sim \operatorname{Beta}\left(a_{3}, b_{3}\right)$ then these marginal priors can be combined to introduce a joint improved chi square prior as

$$
\begin{gathered}
g\left(\theta_{1}, \theta_{2}, \pi\right) \propto \theta_{1}^{\left(d_{3} / 2\right)-1} \theta_{2}^{\left(d_{4} / 2\right)-1} e^{-0.5\left(\theta_{1}+\theta_{2}\right)} \pi^{a_{3}-1}(1-\pi)^{b_{3}-1} \\
\theta_{1}, \theta_{2}>0,0<\pi<1, a_{3}, b_{3}, d_{3}, d_{4}>0
\end{gathered}
$$

where $a_{3}, b_{3}, d_{3}$ and $d_{4}$ are hyper-parameters. Using the prior distributions presented in Equations (3.1)-(3.6) and the likelihood function in Equation (2.7), the posterior distributions can respectively be obtained as

$$
\begin{aligned}
& h_{1}\left(\theta_{1}, \theta_{2}, \pi \mid \mathrm{x}\right) \propto \sum_{k_{1}=0}^{r_{1}-1} \sum_{k_{2}=0}^{r_{2}-1} \sum_{k_{3}=0}^{n-s} \sum_{k_{4}=0}^{k_{3}} \prod_{w=1}^{2}(-1)^{k_{3}}\left(\begin{array}{c}
r_{1}-1 \\
k_{1}
\end{array}\right)\left(\begin{array}{c}
r_{2}-1 \\
k_{2}
\end{array}\right)\left(\begin{array}{c}
n-s \\
k_{3}
\end{array}\right)\left(\begin{array}{c}
k_{3} \\
k_{4}
\end{array}\right) \\
& \times \pi^{k_{1}+k_{3}+k_{4}-\frac{1}{2}}(1-\pi)^{r_{1}+r_{2}+k_{3}-k_{1}-k_{2}-k_{4}-\frac{5}{2}} \theta_{w}^{m_{w}-1} e^{-\theta_{w} \Omega\left(x_{w i}\right)}, \\
& h_{1}\left(\theta_{1}, \theta_{2}, \pi \mid \mathrm{x}\right) \propto \sum_{k_{1}=0}^{r_{1}-1} \sum_{k_{2}=0}^{r_{2}-1} \sum_{k_{3}=0}^{n-s} \sum_{k_{4}=0}^{k_{3}} \prod_{w=1}^{2}(-1)^{k_{3}}\left(\begin{array}{c}
r_{1}-1 \\
k_{1}
\end{array}\right)\left(\begin{array}{c}
r_{2}-1 \\
k_{2}
\end{array}\right)\left(\begin{array}{c}
n-s \\
k_{3}
\end{array}\right)\left(\begin{array}{c}
k_{3} \\
k_{4}
\end{array}\right) \\
& \times \pi^{k_{1}+k_{3}+k_{4}-a_{3}-1}(1-\pi)^{r_{1}+r_{2}+k_{3}-k_{1}-k_{2}-k_{4}-2+b_{3}-1} \theta_{w}^{m_{w}+a_{w}-1} \\
& \times e^{-\theta_{w}\left\{\Omega\left(x_{w i}\right)+b_{w}\right\}}, \\
& h_{1}\left(\theta_{1}, \theta_{2}, \pi \mid \mathrm{x}\right) \propto \sum_{k_{1}=0}^{r_{1}-1} \sum_{k_{2}=0}^{r_{2}-1} \sum_{k_{3}=0}^{n-s} \sum_{k_{4}=0}^{k_{3}} \prod_{w=1}^{2}(-1)^{k_{3}}\left(\begin{array}{c}
r_{1}-1 \\
k_{1}
\end{array}\right)\left(\begin{array}{c}
r_{2}-1 \\
k_{2}
\end{array}\right)\left(\begin{array}{c}
n-s \\
k_{3}
\end{array}\right)\left(\begin{array}{c}
k_{3} \\
k_{4}
\end{array}\right) \\
& \times \pi^{k_{1}+k_{3}+k_{4}-a_{3}-1}(1-\pi)^{r_{1}+r_{2}+k_{3}-k_{1}-k_{2}-k_{4}-2+b_{3}-1} \theta_{w}^{m_{w}+a_{w}-2} \\
& \times e^{-\theta_{w}\left\{\Omega\left(x_{w i}\right)+b_{w}\right\}}, \\
& h_{1}\left(\theta_{1}, \theta_{2}, \pi \mid \mathrm{x}\right) \propto \sum_{k_{1}=0}^{r_{1}-1} \sum_{k_{2}=0}^{r_{2}-1} \sum_{k_{3}=0}^{n-s} \sum_{k_{4}=0}^{k_{3}} \prod_{w=1}^{2}(-1)^{k_{3}}\left(\begin{array}{c}
r_{1}-1 \\
k_{1}
\end{array}\right)\left(\begin{array}{c}
r_{2}-1 \\
k_{2}
\end{array}\right)\left(\begin{array}{c}
n-s \\
k_{3}
\end{array}\right)\left(\begin{array}{c}
k_{3} \\
k_{4}
\end{array}\right) \\
& \times \pi^{k_{1}+k_{3}+k_{4}-a_{3}-1}(1-\pi)^{r_{1}+r_{2}+k_{3}-k_{1}-k_{2}-k_{4}-2+b_{3}-1} \theta_{w}^{m_{w}-0.5} \\
& \times e^{-\theta_{w}\left\{\Omega\left(x_{w i}\right)+0.5 b_{w+3}\right\}}, \\
& h_{1}\left(\theta_{1}, \theta_{2}, \pi \mid \mathrm{x}\right) \propto \sum_{k_{1}=0}^{r_{1}-1} \sum_{k_{2}=0}^{r_{2}-1} \sum_{k_{3}=0}^{n-s} \sum_{k_{4}=0}^{k_{3}} \prod_{w=1}^{2}(-1)^{k_{3}}\left(\begin{array}{c}
r_{1}-1 \\
k_{1}
\end{array}\right)\left(\begin{array}{c}
r_{2}-1 \\
k_{2}
\end{array}\right)\left(\begin{array}{c}
n-s \\
k_{3}
\end{array}\right)\left(\begin{array}{c}
k_{3} \\
k_{4}
\end{array}\right) \\
& \times \pi^{k_{1}+k_{3}+k_{4}-a_{3}-1}(1-\pi)^{r_{1}+r_{2}+k_{3}-k_{1}-k_{2}-k_{4}-2+b_{3}-1} \theta_{w}^{m_{w}} \\
& \times e^{-\theta_{w}\left\{\Omega\left(x_{w i}\right)+d_{w}\right\}}, \\
& h_{1}\left(\theta_{1}, \theta_{2}, \pi \mid \mathrm{x}\right) \propto \sum_{k_{1}=0}^{r_{1}-1} \sum_{k_{2}=0}^{r_{2}-1} \sum_{k_{3}=0}^{n-s} \sum_{k_{4}=0}^{k_{3}} \prod_{w=1}^{2}(-1)^{k_{3}}\left(\begin{array}{c}
r_{1}-1 \\
k_{1}
\end{array}\right)\left(\begin{array}{c}
r_{2}-1 \\
k_{2}
\end{array}\right)\left(\begin{array}{c}
n-s \\
k_{3}
\end{array}\right)\left(\begin{array}{c}
k_{3} \\
k_{4}
\end{array}\right) \\
& \times \pi^{k_{1}+k_{3}+k_{4}-a_{3}-1}(1-\pi)^{r_{1}+r_{2}+k_{3}-k_{1}-k_{2}-k_{4}-2+b_{3}-1} \theta_{w}^{m_{w}+0.5 d_{w+2}} \\
& \times e^{-\theta w\left\{\Omega\left(x_{w i}\right)+0.5\right\}} \text {. }
\end{aligned}
$$


The posterior distributions given in Equations (3.7) and (3.12) be combined to a single equation as

$$
\begin{aligned}
h_{1}\left(\theta_{1}, \theta_{2}, \pi \mid \mathrm{x}\right) & \propto \sum_{k_{1}=0}^{r_{1}-1} \sum_{k_{2}=0}^{r_{2}-1} \sum_{k_{3}=0}^{n-s} \sum_{k_{4}=0}^{k_{3}} \prod_{w=1}^{2}(-1)^{k_{3}}\left(\begin{array}{c}
r_{1}-1 \\
k_{1}
\end{array}\right)\left(\begin{array}{c}
r_{2}-1 \\
k_{2}
\end{array}\right)\left(\begin{array}{c}
n-s \\
k_{3}
\end{array}\right)\left(\begin{array}{c}
k_{3} \\
k_{4}
\end{array}\right) \\
& \times \pi^{k_{1}+k_{3}+k_{4}-l_{1}\left(a_{3}-1\right)-\frac{1}{2}}(1-\pi)^{r_{1}+r_{2}+k_{3}-k_{1}-k_{2}-k_{4}+l_{1}\left(b_{3}-1\right)-\frac{5}{2}} \\
& \times \theta_{w}^{m_{w}-l_{2}+l_{3}\left(a_{w}-1-l_{4}\right)-0.5 l_{5}+l_{7}\left(0.5 d_{2+w}-1\right)} \\
& \times e^{-\theta}\left\{\Omega\left(x_{w i}\right)+l_{3} b_{w}+0.5 l_{5} b_{w+3}+l_{6} d_{w}+0.5 l_{7}\right\}
\end{aligned}
$$

Here, the posterior distributions given in Equations (3.7)-(3.12) can be derived as a special case of the above density Equation (3.13) by putting $l_{1}=l_{2}=l_{3}=l_{4}=l_{5}=l_{6}=l_{7}=0$; $l_{1}=l_{3}=1, l_{2}=l_{4}=l_{5}=l_{6}=l_{7}=0 ; l_{1}=l_{3}=1, l_{2}=l_{4}=l_{5}=l_{6}=l_{7}=0 ;$ $l_{1}=l_{5}=1, l_{2}=l_{3}=l_{4}=l_{6}=l_{7}=0 ; l_{1}=l_{6}=1, l_{2}=l_{3}=l_{4}=l_{5}=l_{7}=0 ;$ and $l_{1}=l_{7}=1, l_{2}=l_{3}=l_{4}=l_{5}=l_{6}=0$, respectively.

Let $A_{1}=k_{1}+k_{3}+k_{4}+l_{1}\left(a_{3}-1\right)-1 / 2, A_{2}=r_{1}+r_{2}+k_{3}-k_{1}-k_{2}-k_{4}+l_{1}\left(b_{3}-1\right)-5 / 2$, $M_{w}=m_{w}-l_{2}+l_{3}\left(a_{w}-1-l_{4}\right)-0.5 l_{5}+l_{7}\left(0.5 d_{2+w}-1\right)$ and $\phi\left(x_{w i}\right)=\Omega\left(x_{w i}\right)+l_{3} b_{w}+$ $0.5 l_{5} b_{w+3}+l_{6} d_{w}+0.5 l_{7}$. Therefore, the model given in Equation (3.13) can be presented as

$$
\begin{aligned}
h\left(\theta_{1}, \theta_{2}, \pi \mid \mathrm{x}\right) & \propto \sum_{k_{1}=0}^{r_{1}-1} \sum_{k_{2}=0}^{r_{2}-1} \sum_{k_{3}=0}^{n-s} \sum_{k_{4}=0}^{k_{3}} \prod_{w=1}^{2}(-1)^{k_{3}}\left(\begin{array}{c}
r_{1}-1 \\
k_{1}
\end{array}\right)\left(\begin{array}{c}
r_{2}-1 \\
k_{2}
\end{array}\right)\left(\begin{array}{c}
n-s \\
k_{3}
\end{array}\right)\left(\begin{array}{c}
k_{3} \\
k_{4}
\end{array}\right) \\
& \times \pi^{A_{1}}(1-\pi)^{A_{2}} \theta_{w}^{M_{w}} \exp \left\{-\theta_{w} \phi\left(x_{w i}\right)\right\} .
\end{aligned}
$$

Consider that

$$
\begin{aligned}
& \int_{0}^{\infty} \int_{0}^{1} \sum_{k_{1}=0}^{r_{1}-1} \sum_{k_{2}=0}^{r_{2}-1} \sum_{k_{3}=0}^{n-s} \sum_{k_{4}=0}^{k_{3}} \prod_{w=1}^{2}(-1)^{k_{3}}\left(\begin{array}{c}
r_{1}-1 \\
k_{1}
\end{array}\right)\left(\begin{array}{c}
r_{2}-1 \\
k_{2}
\end{array}\right)\left(\begin{array}{c}
n-s \\
k_{3}
\end{array}\right)\left(\begin{array}{c}
k_{3} \\
k_{4}
\end{array}\right) \pi^{A_{1}}(1-\pi)^{A_{2}} \theta_{w}^{M_{w}} e^{-\theta_{w} \phi\left(x_{w i}\right)} d \pi d \theta_{1} d \theta_{2} \\
& =\sum_{k_{1}=0}^{r_{1}-1} \sum_{k_{2}=0}^{r_{2}-1} \sum_{k_{3}=0}^{n-s} \sum_{k_{4}=0}^{k_{3}} \prod_{w=1}^{2}(-1)^{k_{3}}\left(\begin{array}{c}
r_{1}-1 \\
k_{1}
\end{array}\right)\left(\begin{array}{c}
r_{2}-1 \\
k_{2}
\end{array}\right)\left(\begin{array}{c}
n-s \\
k_{3}
\end{array}\right)\left(\begin{array}{c}
k_{3} \\
k_{4}
\end{array}\right) \times \int_{0}^{\infty} \int_{0}^{\infty} \int_{0}^{\infty} \pi^{A_{1}}(1-\pi)^{A_{2}} \theta_{w}^{M_{w}} e^{-\theta_{w} \phi\left(x_{w i}\right)} d \pi d \theta_{1} d \theta_{2} \\
& =\sum_{k_{1}=0}^{r_{1}-1} \sum_{k_{2}=0}^{r_{2}-1} \sum_{k_{3}=0}^{n-s} \sum_{k_{4}=0}^{k_{3}} \prod_{w=1}^{2}(-1)^{k_{3}}\left(\begin{array}{c}
r_{1}-1 \\
k_{1}
\end{array}\right)\left(\begin{array}{c}
r_{2}-1 \\
k_{2}
\end{array}\right)\left(\begin{array}{c}
n-s \\
k_{3}
\end{array}\right)\left(\begin{array}{c}
k_{3} \\
k_{4}
\end{array}\right) \frac{B\left(A_{1}+1, A_{2}+1\right) \Gamma\left(M_{w}+1\right)}{\left\{\phi\left(x_{w i}\right)\right\}^{M_{w}+1}},
\end{aligned}
$$

where $B(x, y)$ is a standard Beta function and $\Gamma(x)$ is the gamma function. Therefore the complete density for the posterior distributions given in Equation (3.14) can be obtained as

$$
h\left(\theta_{1}, \theta_{2}, \pi \mid \mathrm{x}\right)=\frac{\sum_{k_{1}=0}^{r_{1}-1} \sum_{k_{2}=0}^{r_{2}-1} \sum_{k_{3}=0}^{n-s} \sum_{k_{4}=0}^{k_{3}} \prod_{w=1}^{2}(-1)^{k_{3}}\left(\begin{array}{c}
r_{1}-1 \\
k_{1}
\end{array}\right)\left(\begin{array}{c}
r_{2}-1 \\
k_{2}
\end{array}\right)\left(\begin{array}{c}
n-s \\
k_{3}
\end{array}\right)\left(\begin{array}{c}
k_{3} \\
k_{4}
\end{array}\right) \pi^{A_{1}}(1-\pi)^{A_{2}} \theta_{w}^{M_{w}} \exp \left\{-\theta_{w} \phi\left(x_{w i}\right)\right\}}{\sum_{k_{1}=0}^{r_{1}-1} \sum_{k_{2}=0}^{r_{2}-1} \sum_{k_{3}=0}^{n-s} \sum_{k_{4}=0}^{k_{3}} \prod_{w=1}^{2}\left(\begin{array}{c}
r_{1}-1 \\
k_{1}
\end{array}\right)\left(\begin{array}{c}
r_{2}-1 \\
k_{2}
\end{array}\right)\left(\begin{array}{c}
n-s \\
k_{3}
\end{array}\right)\left(\begin{array}{c}
k_{3} \\
k_{4}
\end{array}\right) \frac{B\left(A_{1}+1, A_{2}+1\right) \Gamma\left(M_{w}+1\right)}{\left\{\phi\left(x_{w i}\right)\right\}^{M_{w}+1}}} .
$$

Let

$$
D=\sum_{k_{1}=0}^{r_{1}-1} \sum_{k_{2}=0}^{r_{2}-1} \sum_{k_{3}=0}^{n-s} \sum_{k_{4}=0}^{k_{3}} \prod_{w=1}^{2}\left(\begin{array}{c}
r_{1}-1 \\
k_{1}
\end{array}\right)\left(\begin{array}{c}
r_{2}-1 \\
k_{2}
\end{array}\right)\left(\begin{array}{c}
n-s \\
k_{3}
\end{array}\right)\left(\begin{array}{c}
k_{3} \\
k_{4}
\end{array}\right) \frac{B\left(A_{1}+1, A_{2}+1\right) \Gamma\left(M_{w}+1\right)}{\left\{\phi\left(x_{w i}\right)\right\}^{M_{w}+1}} .
$$

Therefore, Equation (3.15) can be presented as

$$
\begin{aligned}
h\left(\theta_{1}, \theta_{2}, \pi \mid \mathrm{x}\right) & =\frac{1}{D} \sum_{k_{1}=0}^{r_{1}-1} \sum_{k_{2}=0}^{r_{2}-1} \sum_{k_{3}=0}^{n-s} \sum_{k_{4}=0}^{k_{3}} \prod_{w=1}^{2}(-1)^{k_{3}}\left(\begin{array}{c}
r_{1}-1 \\
k_{1}
\end{array}\right)\left(\begin{array}{c}
r_{2}-1 \\
k_{2}
\end{array}\right)\left(\begin{array}{c}
n-s \\
k_{3}
\end{array}\right)\left(\begin{array}{c}
k_{3} \\
k_{4}
\end{array}\right) \\
& \times \pi^{A_{1}}(1-\pi)^{A_{2}} \theta_{w}^{M_{w}} e^{-\theta_{w} \phi\left(x_{w i}\right)} .
\end{aligned}
$$


The graphs for the marginal posterior distributions for the parameters of the mixture density, given in Equation (2.3), under different priors are presented in Figures 1 and 2. The graphs are based on the simulated data from the mixture model using a sample of size 50. The legends in the graphs contain following abbreviations: NIP: Non-informative prior; GP: Gamma prior; JGP: Jeffreys Gamma prior; ILP: Inverse levy prior; EP: Exponential prior; CSP: Chi square prior.

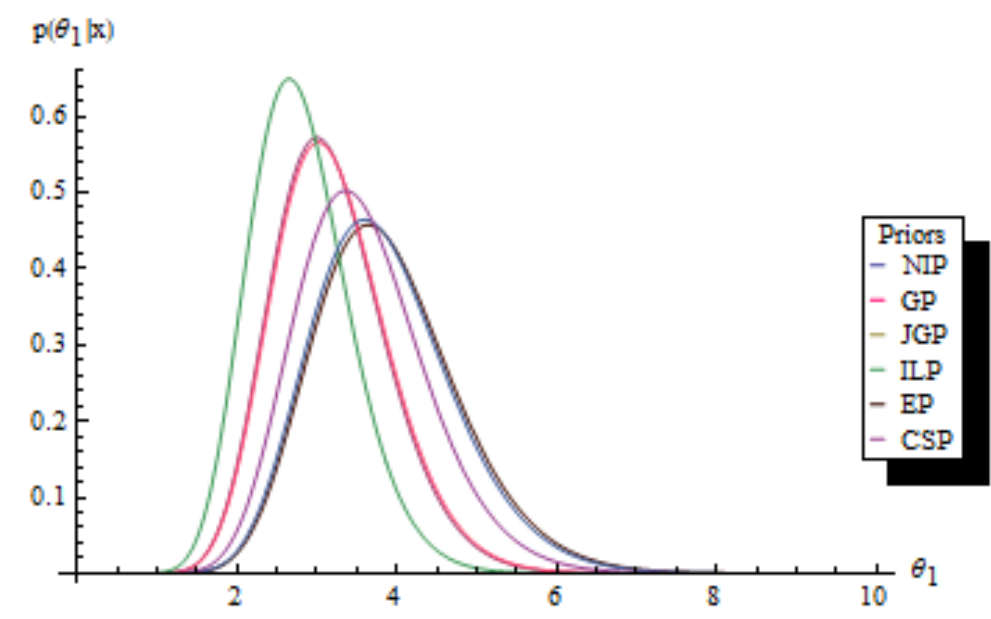

Figure 1. Graph for the marginal posterior distribution of $\theta_{1}$ using different priors.

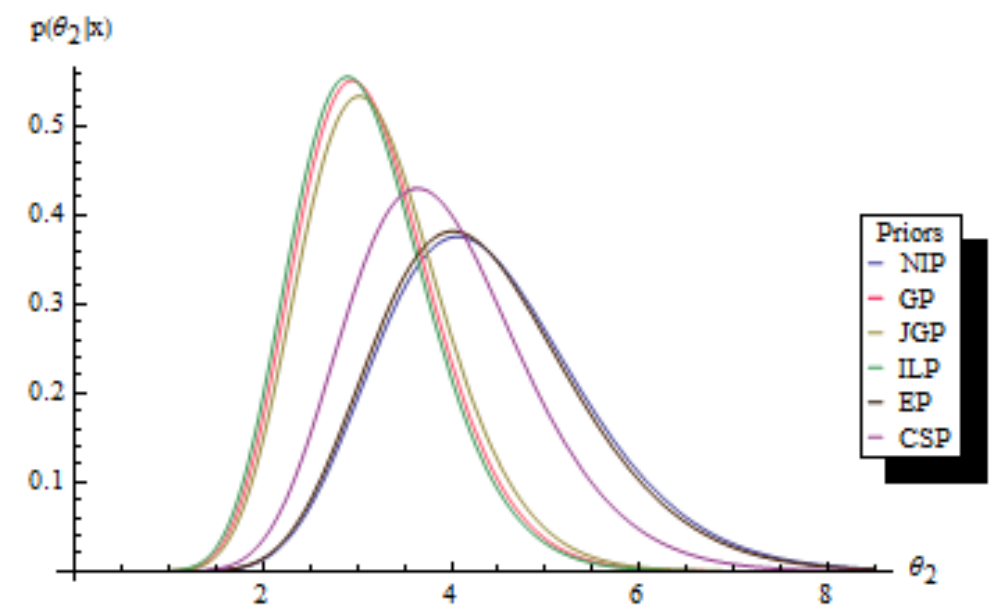

Figure 2. Graph for the marginal posterior distribution of $\theta_{2}$ using different priors.

The graphs indicate that the marginal posterior distributions for the parameters $\theta_{1}$ and $\theta_{2}$ are slightly positively skewed. The tendency of skewness is higher for the second component. In case of first component, it can be observed that height of posterior distribution under inverse levy prior (ILP) is the most. The shape of posterior distributions under gamma and Jeffreys gamma priors are very close to each other. Similarly, the shape of posterior distributions under non-informative prior and the exponential priors are alike. In case second component, the posterior distributions under non-informative prior, gamma prior and Jeffreys gamma prior are having similar shapes. Finally, the posterior distributions based on exponential prior and chi square prior are identical. 


\section{Loss functions and Bayes estimators}

The decision theory suggests that in order to select the best estimator, a loss function must be specified and used to estimate the risk associated with each of the possible estimates. Since, there is no definite analytical process that allows us to identify the proper loss function to be used, most of the analysts uses some symmetric loss functions, these associate equal importance to the losses due to overestimation and underestimation of equal magnitude and obtain the posterior mean as Bayesian estimate. In situations where the loss is asymmetric, we have to employ some asymmetric loss functions. Therefore, we have included both symmetric and asymmetric loss functions for posterior estimation. The description of the both loss function is given as the followings.

\subsection{Squared error loss function (SELF)}

The squared error loss function proposed by [14] and [22] is defined as

$$
L\left(\theta, \theta_{S E L F}\right)=\left(\theta-\theta_{S E L F}\right)^{2} .
$$

The Bayes estimator under this loss function is

$$
\theta_{S E L F}=E(\theta),
$$

where $\theta$ is the parameter. The risk associated with this loss function can be viewed as variance of the estimator and is related to classical least square theory.

\subsection{Squared logarithmic loss function (SLLF)}

Another loss function due to Brown [4] is called squared logarithmic loss function. It can be defined as

$$
L\left(\theta_{S L L F}, \theta\right)=\left(\log \theta_{S L L F}-\log \theta\right)^{2} .
$$

The Bayes estimate under SLLF is

$$
\theta_{S L L F}=\exp \{\mathrm{E}(\log \theta)\} .
$$

The generalized expressions for Bayes estimators and posterior risks under these loss functions have been presented in the following. as

The Bayes estimator of parameter under SELF, denoted by $\left(\hat{\theta}_{1}\right)_{S E L F}$, can be obtained

$$
\begin{aligned}
\left(\hat{\theta}_{1}\right)_{S E L F}=\mathrm{E}\left(\theta_{1}\right)= & \frac{1}{D} \sum_{k_{1}=0}^{r_{1}-1} \sum_{k_{2}=0}^{r_{2}-1} \sum_{k_{3}=0}^{n-s} \sum_{k_{4}=0}^{k_{3}}(-1)^{k_{3}}\left(\begin{array}{c}
r_{1}-1 \\
k_{1}
\end{array}\right)\left(\begin{array}{c}
r_{2}-1 \\
k_{2}
\end{array}\right)\left(\begin{array}{c}
n-s \\
k_{3}
\end{array}\right)\left(\begin{array}{c}
k_{3} \\
k_{4}
\end{array}\right) \\
& \times \int_{0}^{\infty} \int_{0}^{\infty} \int_{0}^{1} \pi^{A_{1}}(1-\pi)^{A_{2}} \theta_{1}^{M_{1}+1} \theta_{2}^{M_{2}} \exp \left\{-\theta_{1} \phi\left(x_{1 i}\right)\right\} \\
& \times \exp \left\{-\theta_{2} \phi\left(x_{2 i}\right)\right\} d \pi d \theta_{1} d \theta_{2} \\
= & \frac{1}{D} \sum_{k_{1}=0}^{r_{1}-1} \sum_{k_{2}=0}^{r_{2}-1} \sum_{k_{3}=0}^{n-s} \sum_{k_{4}=0}^{k_{3}}(-1)^{k_{3}}\left(\begin{array}{c}
r_{1}-1 \\
k_{1}
\end{array}\right)\left(\begin{array}{c}
r_{2}-1 \\
k_{2}
\end{array}\right)\left(\begin{array}{c}
n-s \\
k_{3}
\end{array}\right)\left(\begin{array}{c}
k_{3} \\
k_{4}
\end{array}\right) \\
& \times \frac{B\left(A_{1}+1, A_{2}+1\right) \Gamma\left(M_{1}+2\right) \Gamma\left(M_{2}+1\right)}{\left\{\phi\left(x_{1 i}\right)\right\}^{M_{1}+2}\left\{\phi\left(x_{2 i}\right)\right\}^{M_{2}+1}} .
\end{aligned}
$$


The Bayes estimator of parameter $\theta_{2}$ under SELF, denoted by $\left(\hat{\theta}_{2}\right)_{S E L F}$, can be derived as

$$
\begin{aligned}
\left(\hat{\theta}_{2}\right)_{S E L F}=\mathrm{E}\left(\theta_{1}\right)= & \frac{1}{D} \sum_{k_{1}=0}^{r_{1}-1} \sum_{k_{2}=0}^{r_{2}-1} \sum_{k_{3}=0}^{n-s} \sum_{k_{4}=0}^{k_{3}}(-1)^{k_{3}}\left(\begin{array}{c}
r_{1}-1 \\
k_{1}
\end{array}\right)\left(\begin{array}{c}
r_{2}-1 \\
k_{2}
\end{array}\right)\left(\begin{array}{c}
n-s \\
k_{3}
\end{array}\right)\left(\begin{array}{c}
k_{3} \\
k_{4}
\end{array}\right) \\
& \times \int_{0}^{\infty} \int_{0}^{\infty} \int_{0}^{1} \pi^{A_{1}}(1-\pi)^{A_{2}} \theta_{1}^{M_{1}+1} \theta_{2}^{M_{2}+1} \exp \left\{-\theta_{1} \phi\left(x_{1 i}\right)\right\} \\
& \times \exp \left\{-\theta_{2} \phi\left(x_{2 i}\right)\right\} d \pi d \theta_{1} d \theta_{2} \\
= & \frac{1}{D} \sum_{k_{1}=0}^{r_{1}-1} \sum_{k_{2}=0}^{r_{2}-1} \sum_{k_{3}=0}^{n-s} \sum_{k_{4}=0}^{k_{3}}(-1)^{k_{3}}\left(\begin{array}{c}
r_{1}-1 \\
k_{1}
\end{array}\right)\left(\begin{array}{c}
r_{2}-1 \\
k_{2}
\end{array}\right)\left(\begin{array}{c}
n-s \\
k_{3}
\end{array}\right)\left(\begin{array}{c}
k_{3} \\
k_{4}
\end{array}\right) \\
& \times \frac{B\left(A_{1}+2, A_{2}+1\right) \Gamma\left(M_{1}+1\right) \Gamma\left(M_{2}+1\right)}{\left\{\phi\left(x_{1 i}\right)\right\}^{M_{1}+1}\left\{\phi\left(x_{2 i}\right)\right\}^{M_{2}+1}} .
\end{aligned}
$$

The Bayes estimator of parameter $\pi$ under SELF, denoted by $(\hat{\pi})_{S E L F}$, can be obtained as

$$
\begin{aligned}
\left(\hat{\pi}_{2}\right)_{S E L F}= & \mathrm{E}(\pi)=\frac{1}{D} \sum_{k_{1}=0}^{r_{1}-1} \sum_{k_{2}=0}^{r_{2}-1} \sum_{k_{3}=0}^{n-s} \sum_{k_{4}=0}^{k_{3}}(-1)^{k_{3}}\left(\begin{array}{c}
r_{1}-1 \\
k_{1}
\end{array}\right)\left(\begin{array}{c}
r_{2}-1 \\
k_{2}
\end{array}\right)\left(\begin{array}{c}
n-s \\
k_{3}
\end{array}\right)\left(\begin{array}{c}
k_{3} \\
k_{4}
\end{array}\right) \\
& \times \int_{0}^{\infty} \int_{0}^{\infty} \int_{0}^{1} \pi^{A_{1}+1}(1-\pi)^{A_{2}} \theta_{1}^{M_{1}} \theta_{2}^{M_{2}} \exp \left\{-\theta_{1} \phi\left(x_{1 i}\right)\right\} \\
& \times \exp \left\{-\theta_{2} \phi\left(x_{2 i}\right)\right\} d \pi d \theta_{1} d \theta_{2} \\
= & \frac{1}{D} \sum_{k_{1}=0}^{r_{1}-1} \sum_{k_{2}=0}^{r_{2}-1} \sum_{k_{3}=0}^{n-s} \sum_{k_{4}=0}^{k_{3}}(-1)^{k_{3}}\left(\begin{array}{c}
r_{1}-1 \\
k_{1}
\end{array}\right)\left(\begin{array}{c}
r_{2}-1 \\
k_{2}
\end{array}\right)\left(\begin{array}{c}
n-s \\
k_{3}
\end{array}\right)\left(\begin{array}{c}
k_{3} \\
k_{4}
\end{array}\right) \\
& \times \frac{B\left(A_{1}+2, A_{2}+1\right) \Gamma\left(M_{1}+1\right) \Gamma\left(M_{2}+1\right)}{\left\{\phi\left(x_{1 i}\right)\right\}^{M_{1}+1}\left\{\phi\left(x_{2 i}\right)\right\}^{M_{2}+1}} .
\end{aligned}
$$

Similarly, the generalized expressions of the estimator for the posterior risk under SELF, using the formula $\rho(\theta)_{S E L F}=\mathrm{E}\left(\theta^{2}\right)-\{\mathrm{E}(\theta)\}^{2}$, have been presented in the followings.

The Bayes estimator of posterior risk for parameter $\theta_{1}$ under SELF, denoted by $\rho\left(\theta_{1}\right)_{S E L F}$, can be obtained as

$$
\begin{aligned}
\rho\left(\theta_{1}\right)_{S E L F}= & \frac{1}{D} \sum_{k_{1}=0}^{r_{1}-1} \sum_{k_{2}=0}^{r_{2}-1} \sum_{k_{3}=0}^{n-s} \sum_{k_{4}=0}^{k_{3}}(-1)^{k_{3}}\left(\begin{array}{c}
r_{1}-1 \\
k_{1}
\end{array}\right)\left(\begin{array}{c}
r_{2}-1 \\
k_{2}
\end{array}\right)\left(\begin{array}{c}
n-s \\
k_{3}
\end{array}\right)\left(\begin{array}{c}
k_{3} \\
k_{4}
\end{array}\right) \\
& \times \frac{B\left(A_{1}+1, A_{2}+1\right) \Gamma\left(M_{1}+3\right) \Gamma\left(M_{2}+1\right)}{\left\{\phi\left(x_{1 i}\right)\right\}^{M_{1}+3}\left\{\phi\left(x_{2 i}\right)\right\}^{M_{2}+1}} \\
& -\left[\frac{1}{D} \sum_{k_{1}=0}^{r_{1}-1} \sum_{k_{2}=0}^{r_{2}-1} \sum_{k_{3}=0}^{n-s} \sum_{k_{4}=0}^{k_{3}}(-1)^{k_{3}}\left(\begin{array}{c}
r_{1}-1 \\
k_{1}
\end{array}\right)\left(\begin{array}{c}
r_{2}-1 \\
k_{2}
\end{array}\right)\left(\begin{array}{c}
n-s \\
k_{3}
\end{array}\right)\left(\begin{array}{c}
k_{3} \\
k_{4}
\end{array}\right)\right. \\
& \left.\times \frac{B\left(A_{1}+1, A_{2}+1\right) \Gamma\left(M_{1}+2\right) \Gamma\left(M_{2}+1\right)}{\left\{\phi\left(x_{1 i}\right)\right\}^{M_{1}+2}\left\{\phi\left(x_{2 i}\right)\right\}^{M_{2}+1}}\right]^{2} .
\end{aligned}
$$


The Bayes estimator of posterior risk for parameter $\theta_{2}$ under SELF, denoted by $\rho\left(\theta_{2}\right)_{S E L F}$, can be obtained as

$$
\begin{aligned}
\rho\left(\theta_{2}\right)_{\text {SELF }}= & \frac{1}{D} \sum_{k_{1}=0}^{r_{1}-1} \sum_{k_{2}=0}^{r_{2}-1} \sum_{k_{3}=0}^{n-s} \sum_{k_{4}=0}^{k_{3}}(-1)^{k_{3}}\left(\begin{array}{c}
r_{1}-1 \\
k_{1}
\end{array}\right)\left(\begin{array}{c}
r_{2}-1 \\
k_{2}
\end{array}\right)\left(\begin{array}{c}
n-s \\
k_{3}
\end{array}\right)\left(\begin{array}{l}
k_{3} \\
k_{4}
\end{array}\right) \\
& \times \frac{B\left(A_{1}+1, A_{2}+1\right) \Gamma\left(M_{1}+1\right) \Gamma\left(M_{2}+3\right)}{\left\{\phi\left(x_{1 i}\right)\right\}^{M_{1}+1}\left\{\phi\left(x_{2 i}\right)\right\}^{M_{2}+3}} \\
& -\left[\frac{1}{D} \sum_{k_{1}=0}^{r_{1}-1} \sum_{k_{2}=0}^{r_{2}-1} \sum_{k_{3}=0}^{n-s} \sum_{k_{4}=0}^{k_{3}}(-1)^{k_{3}}\left(\begin{array}{c}
r_{1}-1 \\
k_{1}
\end{array}\right)\left(\begin{array}{c}
r_{2}-1 \\
k_{2}
\end{array}\right)\left(\begin{array}{c}
n-s \\
k_{3}
\end{array}\right)\left(\begin{array}{c}
k_{3} \\
k_{4}
\end{array}\right)\right. \\
& \left.\times \frac{B\left(A_{1}+1, A_{2}+1\right) \Gamma\left(M_{1}+1\right) \Gamma\left(M_{2}+2\right)}{\left\{\phi\left(x_{1 i}\right)\right\}^{M_{1}+2}\left\{\phi\left(x_{2 i}\right)\right\}^{M_{2}+1}}\right]^{2} .
\end{aligned}
$$

Similarly, the Bayes estimator of posterior risk for parameter under SELF, denoted by $\rho(\pi)_{S E L F}$, can be presented as

$$
\begin{aligned}
\rho(\pi)_{S E L F}= & \frac{1}{D} \sum_{k_{1}=0}^{r_{1}-1} \sum_{k_{2}=0}^{r_{2}-1} \sum_{k_{3}=0}^{n-s} \sum_{k_{4}=0}^{k_{3}}(-1)^{k_{3}}\left(\begin{array}{c}
r_{1}-1 \\
k_{1}
\end{array}\right)\left(\begin{array}{c}
r_{2}-1 \\
k_{2}
\end{array}\right)\left(\begin{array}{c}
n-s \\
k_{3}
\end{array}\right)\left(\begin{array}{c}
k_{3} \\
k_{4}
\end{array}\right) \\
& \times \frac{B\left(A_{1}+1, A_{2}+1\right) \Gamma\left(M_{1}+3\right) \Gamma\left(M_{2}+1\right)}{\left\{\phi\left(x_{1 i}\right)\right\}^{M_{1}+1}\left\{\phi\left(x_{2 i}\right)\right\}^{M_{2}+1}} \\
& -\left[\frac{1}{D} \sum_{k_{1}=0}^{r_{1}-1} \sum_{k_{2}=0}^{r_{2}-1} \sum_{k_{3}=0}^{n-s} \sum_{k_{4}=0}^{k_{3}}(-1)^{k_{3}}\left(\begin{array}{c}
r_{1}-1 \\
k_{1}
\end{array}\right)\left(\begin{array}{c}
r_{2}-1 \\
k_{2}
\end{array}\right)\left(\begin{array}{c}
n-s \\
k_{3}
\end{array}\right)\left(\begin{array}{c}
k_{3} \\
k_{4}
\end{array}\right)\right. \\
& \left.\times \frac{B\left(A_{1}+1, A_{2}+1\right) \Gamma\left(M_{1}+1\right) \Gamma\left(M_{2}+1\right)}{\left\{\phi\left(x_{1 i}\right)\right\}^{M_{1}+1}\left\{\phi\left(x_{2 i}\right)\right\}^{M_{2}+1}}\right]^{2} .
\end{aligned}
$$

Again, the generalized expression for the Bayes estimators and posterior risks based on SLLF are:

The Bayes estimator of under SLLF, can be obtained by using following formula.

$$
\left(\hat{\theta}_{1}\right)_{S L L F}=\exp \left\{\mathrm{E}\left(\log \theta_{1}\right)\right\}
$$

Consider that

$$
\begin{aligned}
\mathrm{E}\left(\log \theta_{1}\right)= & \frac{1}{D} \sum_{k_{1}=0}^{r_{1}-1} \sum_{k_{2}=0}^{r_{2}-1} \sum_{k_{3}=0}^{n-s} \sum_{k_{4}=0}^{k_{3}}(-1)^{k_{3}}\left(\begin{array}{c}
r_{1}-1 \\
k_{1}
\end{array}\right)\left(\begin{array}{c}
r_{2}-1 \\
k_{2}
\end{array}\right)\left(\begin{array}{c}
n-s \\
k_{3}
\end{array}\right)\left(\begin{array}{c}
k_{3} \\
k_{4}
\end{array}\right) \\
& \times \int_{0}^{\infty} \int_{0}^{\infty} \int_{0}^{1} \pi^{A_{1}}(1-\pi)^{A_{2}} \theta_{1}^{M_{1}+1} \theta_{2}^{M_{2}} \exp \left\{-\log \theta_{1} \phi\left(x_{1 i}\right)\right\} \\
& \times \exp \left\{-\theta_{2} \phi\left(x_{2 i}\right)\right\} d \pi d \theta_{1} d \theta_{2} .
\end{aligned}
$$

Since $\int_{0}^{\infty} \log (x) x^{a-1} e^{-b x} d x=\frac{\Gamma(a)[\psi(a)-\log (b)]}{b^{a}}$,

$$
\begin{aligned}
\mathrm{E}\left(\log \theta_{1}\right)= & \frac{1}{D} \sum_{k_{1}=0}^{r_{1}-1} \sum_{k_{2}=0}^{r_{2}-1} \sum_{k_{3}=0}^{n-s} \sum_{k_{4}=0}^{k_{3}} \prod_{w=1}^{2}(-1)^{k_{3}}\left(\begin{array}{c}
r_{1}-1 \\
k_{1}
\end{array}\right)\left(\begin{array}{c}
r_{2}-1 \\
k_{2}
\end{array}\right)\left(\begin{array}{c}
n-s \\
k_{3}
\end{array}\right)\left(\begin{array}{c}
k_{3} \\
k_{4}
\end{array}\right) \\
& \times \frac{B\left(A_{1}+1, A_{2}+1\right)\left(T_{1}\right) \Gamma\left(M_{w}+1\right)}{\left\{\phi\left(x_{w i}\right)\right\}^{M_{w}+1}}
\end{aligned}
$$


where $T_{1}=\psi\left(M_{1}+1\right)-\ln \left\{\phi\left(x_{1 i}\right)\right\}$ and $\psi(x)$ is digamma function. Hence, the Bayes estimator for $\theta_{1}$ under SLLF, denoted by $\left(\hat{\theta}_{1}\right)_{S L L F}$, can be written as

$$
\begin{aligned}
\left(\hat{\theta}_{1}\right)_{S L L F}= & \exp \left\{\frac{1}{D} \sum_{k_{1}=0}^{r_{1}-1} \sum_{k_{2}=0}^{r_{2}-1} \sum_{k_{3}=0}^{n-s} \sum_{k_{4}=0}^{k_{3}} \prod_{w=1}^{2}(-1)^{k_{3}}\left(\begin{array}{c}
r_{1}-1 \\
k_{1}
\end{array}\right)\left(\begin{array}{c}
r_{2}-1 \\
k_{2}
\end{array}\right)\left(\begin{array}{c}
n-s \\
k_{3}
\end{array}\right)\left(\begin{array}{c}
k_{3} \\
k_{4}
\end{array}\right)\right\} \\
& \times \exp \left\{\frac{B\left(A_{1}+1, A_{2}+1\right)\left(T_{1}\right) \Gamma\left(M_{w}+1\right)}{\left\{\phi\left(x_{w i}\right)\right\}^{M_{w}+1}}\right\}
\end{aligned}
$$

Similarly, the Bayes estimator for $\theta_{2}$ under SLLF, denoted by $\left(\hat{\theta}_{2}\right)_{S L L F}$, can be obtained as

$$
\begin{aligned}
\left(\hat{\theta}_{1}\right)_{S L L F}= & \exp \left\{\frac{1}{D} \sum_{k_{1}=0}^{r_{1}-1} \sum_{k_{2}=0}^{r_{2}-1} \sum_{k_{3}=0}^{n-s} \sum_{k_{4}=0}^{k_{3}} \prod_{w=1}^{2}(-1)^{k_{3}}\left(\begin{array}{c}
r_{1}-1 \\
k_{1}
\end{array}\right)\left(\begin{array}{c}
r_{2}-1 \\
k_{2}
\end{array}\right)\left(\begin{array}{c}
n-s \\
k_{3}
\end{array}\right)\left(\begin{array}{c}
k_{3} \\
k_{4}
\end{array}\right)\right\} \\
& \times \exp \left\{\frac{B\left(A_{1}+1, A_{2}+1\right)\left(T_{2}\right) \Gamma\left(M_{w}+1\right)}{\left\{\phi\left(x_{w i}\right)\right\}^{M_{w}+1}}\right\}
\end{aligned}
$$

where $T_{2}=\psi\left(M_{2}+1\right)-\ln \left\{\phi\left(x_{2 i}\right)\right\}$.

Similarly, the Bayes estimator for $\pi$ under SLLF, denoted by $(\hat{\pi})_{S L L F}=\exp \{\mathrm{E}(\log \pi)\}$, can be obtained as

$$
\begin{aligned}
\mathrm{E}(\log \pi)= & \frac{1}{D} \sum_{k_{1}=0}^{r_{1}-1} \sum_{k_{2}=0}^{r_{2}-1} \sum_{k_{3}=0}^{n-s} \sum_{k_{4}=0}^{k_{3}} \prod_{w=1}^{2}(-1)^{k_{3}\left(\begin{array}{c}
r_{1}-1 \\
k_{1}
\end{array}\right)\left(\begin{array}{c}
r_{2}-1 \\
k_{2}
\end{array}\right)\left(\begin{array}{c}
n-s \\
k_{3}
\end{array}\right)\left(\begin{array}{c}
k_{3} \\
k_{4}
\end{array}\right)} \\
& \times \int_{0}^{\infty} \int_{0}^{\infty} \int_{0}^{1} \log (\pi) \pi^{A_{1}}(1-\pi)^{A_{2}} \theta_{1}^{M_{1}} \theta_{2}^{M_{2}} \exp \left\{-\theta_{1} \phi\left(x_{1 i}\right)\right\} \\
& \times \exp \left\{-\theta_{2} \phi\left(x_{2 i}\right)\right\} d \pi d \theta_{1} d \theta_{2} .
\end{aligned}
$$

Since $\int_{0}^{1} \log (x) x^{a-1}(1-x)^{b-1} d x=B(a, b)\{\psi(a)-\psi(a+b)\}$,

$$
\begin{aligned}
\mathrm{E}(\log \pi) & =\frac{1}{D} \sum_{k_{1}=0}^{r_{1}-1} \sum_{k_{2}=0}^{r_{2}-1} \sum_{k_{3}=0}^{n-s} \sum_{k_{4}=0}^{k_{3}} \prod_{w=1}^{2}(-1)^{k_{3}}\left(\begin{array}{c}
r_{1}-1 \\
k_{1}
\end{array}\right)\left(\begin{array}{c}
r_{2}-1 \\
k_{2}
\end{array}\right)\left(\begin{array}{c}
n-s \\
k_{3}
\end{array}\right)\left(\begin{array}{c}
k_{3} \\
k_{4}
\end{array}\right) \\
& \times \frac{B\left(A_{1}+1, A_{2}+1\right)\left(W_{1}\right) \Gamma\left(M_{w}+1\right)}{\left\{\phi\left(x_{w i}\right)\right\}^{M_{w}+1}}
\end{aligned}
$$

where $W_{1}=\psi\left(A_{1}\right)-\psi\left(A_{1}+A_{2}\right)$ and $\psi(x)$ is the digamma function. Hence, the Bayes estimator for $\pi$ under SLLF, denoted by $(\hat{\pi})_{S L L F}$, can be given as

$$
\begin{aligned}
(\hat{\pi})_{S L L F}= & \exp \left\{\frac{1}{D} \sum_{k_{1}=0}^{r_{1}-1} \sum_{k_{2}=0}^{r_{2}-1} \sum_{k_{3}=0}^{n-s} \sum_{k_{4}=0}^{k_{3}} \prod_{w=1}^{2}(-1)^{k_{3}}\left(\begin{array}{c}
r_{1}-1 \\
k_{1}
\end{array}\right)\left(\begin{array}{c}
r_{2}-1 \\
k_{2}
\end{array}\right)\left(\begin{array}{c}
n-s \\
k_{3}
\end{array}\right)\left(\begin{array}{c}
k_{3} \\
k_{4}
\end{array}\right)\right. \\
& \left.\times \frac{B\left(A_{1}+1, A_{2}+1\right)\left(W_{1}\right) \Gamma\left(M_{w}+1\right)}{\left\{\phi\left(x_{w i}\right)\right\}^{M_{w}+1}}\right\}
\end{aligned}
$$

The formula for the derivation of estimator of posterior risk for the parameter $\theta$ under SLLF is $\rho(\theta)_{S L L F}=\mathrm{E}(\log \theta)^{2}-\{\mathrm{E}(\log \theta)\}^{2}$. 
Accordingly, the Bayes estimator of posterior risk for parameter under SLLF can be obtained as $\rho\left(\theta_{1}\right)_{S L L F}$. Consider that

$$
\begin{aligned}
\mathrm{E}\left(\log \theta_{1}\right)^{2}= & \frac{1}{D} \sum_{k_{1}=0}^{r_{1}-1} \sum_{k_{2}=0}^{r_{2}-1} \sum_{k_{3}=0}^{n-s} \sum_{k_{4}=0}^{k_{3}} \prod_{w=1}^{2}(-1)^{k_{3}\left(\begin{array}{c}
r_{1}-1 \\
k_{1}
\end{array}\right)\left(\begin{array}{c}
r_{2}-1 \\
k_{2}
\end{array}\right)\left(\begin{array}{c}
n-s \\
k_{3}
\end{array}\right)\left(\begin{array}{c}
k_{3} \\
k_{4}
\end{array}\right)} \\
& \times \int_{0}^{\infty} \int_{0}^{\infty} \int_{0}^{1} \pi^{A_{1}}(1-\pi)^{A_{2}}\left\{\log \left(\theta_{1}\right)\right\}^{2} \theta_{1}^{M_{1}} \theta_{2}^{M_{2}} \exp \left\{-\theta_{1} \phi\left(x_{1 i}\right)\right\} \\
& \times \exp \left\{-\theta_{2} \phi\left(x_{2 i}\right)\right\} d \pi d \theta_{1} d \theta_{2} .
\end{aligned}
$$

Since $\int_{0}^{\infty}\{\log (x)\}^{2} x^{a-1} e^{-b x} d x=\frac{\Gamma(a)\left[\{\psi(a)-\log (b)\}^{2}-\psi_{1}(a)\right]}{b^{a}}$,

$$
\begin{aligned}
\mathrm{E}\left(\log \theta_{1}\right)^{2} & =\frac{1}{D} \sum_{k_{1}=0}^{r_{1}-1} \sum_{k_{2}=0}^{r_{2}-1} \sum_{k_{3}=0}^{n-s} \sum_{k_{4}=0}^{k_{3}} \prod_{w=1}^{2}(-1)^{k_{3}}\left(\begin{array}{c}
r_{1}-1 \\
k_{1}
\end{array}\right)\left(\begin{array}{c}
r_{2}-1 \\
k_{2}
\end{array}\right)\left(\begin{array}{c}
n-s \\
k_{3}
\end{array}\right)\left(\begin{array}{c}
k_{3} \\
k_{4}
\end{array}\right) \\
& \times \frac{B\left(A_{1}+1, A_{2}+1\right)\left[\left\{T_{1}\right\}^{2}+\psi_{1}\left(M_{1}+1\right)\right]}{\left\{\Gamma\left(M_{w}+1\right)\right\}^{-1}\left\{\phi\left(x_{w i}\right)\right\}^{M_{w}+1}}
\end{aligned}
$$

where $\psi_{1}(x)$ is a trigamma function. Hence, the estimator for $\theta_{1}$ posterior risk of under SLLF can be given as

$$
\begin{aligned}
\rho\left(\theta_{1}\right)_{S L L F}= & \frac{1}{D} \sum_{k_{1}=0}^{r_{1}-1} \sum_{k_{2}=0}^{r_{2}-1} \sum_{k_{3}=0}^{n-s} \sum_{k_{4}=0}^{k_{3}} \prod_{w=1}^{2}(-1)^{k_{3}}\left(\begin{array}{c}
r_{1}-1 \\
k_{1}
\end{array}\right)\left(\begin{array}{c}
r_{2}-1 \\
k_{2}
\end{array}\right)\left(\begin{array}{c}
n-s \\
k_{3}
\end{array}\right)\left(\begin{array}{c}
k_{3} \\
k_{4}
\end{array}\right) \\
& \times \frac{B\left(A_{1}+1, A_{2}+1\right)\left[\left\{T_{1}\right\}^{2}+\psi_{1}\left(M_{1}+1\right)\right]}{\left\{\Gamma\left(M_{w}+1\right)\right\}^{-1}\left\{\phi\left(x_{w i}\right)\right\}^{M_{w}+1}} \\
& -\left[\frac{1}{D} \sum_{k_{1}=0}^{r_{1}-1} \sum_{k_{2}=0}^{r_{2}-1} \sum_{k_{3}=0}^{n-s} \sum_{k_{4}=0}^{k_{3}} \prod_{w=1}^{2}(-1)^{k_{3}}\left(\begin{array}{c}
r_{1}-1 \\
k_{1}
\end{array}\right)\left(\begin{array}{c}
r_{2}-1 \\
k_{2}
\end{array}\right)\left(\begin{array}{c}
n-s \\
k_{3}
\end{array}\right)\left(\begin{array}{c}
k_{3} \\
k_{4}
\end{array}\right)\right. \\
& \times \frac{B\left(A_{1}+1, A_{2}+1\right)\left(T_{1}\right) \Gamma\left(M_{w}+1\right)}{\left\{\phi\left(x_{w i}\right)\right\}^{M_{w}+1}} .
\end{aligned}
$$

Similarly, by little modifications, the estimator for $\theta_{2}$ posterior risk of under SLLF, denoted by $\rho\left(\theta_{2}\right)_{S L L F}$ can be presented as

$$
\begin{aligned}
\rho\left(\theta_{2}\right)_{S L L F}= & \frac{1}{D} \sum_{k_{1}=0}^{r_{1}-1} \sum_{k_{2}=0}^{r_{2}-1} \sum_{k_{3}=0}^{n-s} \sum_{k_{4}=0}^{k_{3}} \prod_{w=1}^{2}(-1)^{k_{3}}\left(\begin{array}{c}
r_{1}-1 \\
k_{1}
\end{array}\right)\left(\begin{array}{c}
r_{2}-1 \\
k_{2}
\end{array}\right)\left(\begin{array}{c}
n-s \\
k_{3}
\end{array}\right)\left(\begin{array}{c}
k_{3} \\
k_{4}
\end{array}\right) \\
& \times \frac{B\left(A_{1}+1, A_{2}+1\right)\left[\left\{T_{2}\right\}^{2}+\psi_{1}\left(M_{2}+1\right)\right]}{\left\{\Gamma\left(M_{w}+1\right)\right\}^{-1}\left\{\phi\left(x_{w i}\right)\right\}^{M_{w}+1}} \\
& -\left[\frac{1}{D} \sum_{k_{1}=0}^{r_{1}-1} \sum_{k_{2}=0}^{r_{2}-1} \sum_{k_{3}=0}^{n-s} \sum_{k_{4}=0}^{k_{3}} \prod_{w=1}^{2}(-1)^{k_{3}}\left(\begin{array}{c}
r_{1}-1 \\
k_{1}
\end{array}\right)\left(\begin{array}{c}
r_{2}-1 \\
k_{2}
\end{array}\right)\left(\begin{array}{c}
n-s \\
k_{3}
\end{array}\right)\left(\begin{array}{c}
k_{3} \\
k_{4}
\end{array}\right)\right. \\
& \left.\times \frac{B\left(A_{1}+1, A_{2}+1\right)\left(T_{2}\right) \Gamma\left(M_{w}+1\right)}{\left\{\phi\left(x_{w i}\right)\right\}^{M_{w}+1}}\right]^{2} .
\end{aligned}
$$


The Bayes estimator of posterior risk for $\pi$ under SLLF can be derived by using the formula $\rho(\pi)_{S L L F}=\mathrm{E}(\log \pi)^{2}-\{\mathrm{E}(\log \pi)\}^{2}$. Consider that

$$
\begin{aligned}
\mathrm{E}(\log \pi)^{2}= & \frac{1}{D} \sum_{k_{1}=0}^{r_{1}-1} \sum_{k_{2}=0}^{r_{2}-1} \sum_{k_{3}=0}^{n-s} \sum_{k_{4}=0}^{k_{3}} \prod_{w=1}^{2}(-1)^{k_{3}}\left(\begin{array}{c}
r_{1}-1 \\
k_{1}
\end{array}\right)\left(\begin{array}{c}
r_{2}-1 \\
k_{2}
\end{array}\right)\left(\begin{array}{c}
n-s \\
k_{3}
\end{array}\right)\left(\begin{array}{c}
k_{3} \\
k_{4}
\end{array}\right) \\
& \times \int_{0}^{\infty} \int_{0}^{\infty} \int_{0}^{1} \pi^{A_{1}}(1-\pi)^{A_{2}}\{\log (\pi)\}^{2} \theta_{1}^{M_{1}} \theta_{2}^{M_{2}} \\
& \times \exp \left\{-\theta_{1} \phi\left(x_{1 i}\right)\right\} \exp \left\{-\theta_{2} \phi\left(x_{2 i}\right)\right\} d \pi d \theta_{1} d \theta_{2} .
\end{aligned}
$$

Since $\int_{0}^{1}\{\log (x)\}^{2} x^{a-1}(1-x)^{b-1} d x=B(a, b)\left[\{\psi(a)-\psi(a+b)\}^{2}+\left\{\psi_{1}(a)-\psi_{1}(a+b)\right\}\right]$,

$$
\begin{aligned}
\mathrm{E}(\log \pi)^{2} & =\frac{1}{D} \sum_{k_{1}=0}^{r_{1}-1} \sum_{k_{2}=0}^{r_{2}-1} \sum_{k_{3}=0}^{n-s} \sum_{k_{4}=0}^{k_{3}} \prod_{w=1}^{2}(-1)^{k_{3}}\left(\begin{array}{c}
r_{1}-1 \\
k_{1}
\end{array}\right)\left(\begin{array}{c}
r_{2}-1 \\
k_{2}
\end{array}\right)\left(\begin{array}{c}
n-s \\
k_{3}
\end{array}\right)\left(\begin{array}{l}
k_{3} \\
k_{4}
\end{array}\right) \\
& \times \frac{B\left(A_{1}+1, A_{2}+1\right)\left[\left\{W_{1}\right\}^{2}+W_{2}\right]}{\left\{\Gamma\left(M_{w}+1\right)\right\}^{-1}\left\{\phi\left(x_{w i}\right)\right\}^{M_{w}+1}}
\end{aligned}
$$

where $W_{1}=\psi\left(A_{1}\right)-\psi\left(A_{1}+A_{2}\right), W_{2}=\psi_{1}\left(A_{1}\right)-\psi_{1}\left(A_{1}+A_{2}\right) ; B(x, y)$ is a standard beta function; $\psi(x)$ is a digamma function and $\psi_{1}(x)$ is a trigamma function. Hence, the estimator for posterior risk of can be obtained as

$$
\begin{aligned}
\rho\left(\theta_{2}\right)_{S L L F}= & \frac{1}{D} \sum_{k_{1}=0}^{r_{1}-1} \sum_{k_{2}=0}^{r_{2}-1} \sum_{k_{3}=0}^{n-s} \sum_{k_{4}=0}^{k_{3}} \prod_{w=1}^{2}(-1)^{k_{3}}\left(\begin{array}{c}
r_{1}-1 \\
k_{1}
\end{array}\right)\left(\begin{array}{c}
r_{2}-1 \\
k_{2}
\end{array}\right)\left(\begin{array}{c}
n-s \\
k_{3}
\end{array}\right)\left(\begin{array}{c}
k_{3} \\
k_{4}
\end{array}\right) \\
& \times \frac{B\left(A_{1}+1, A_{2}+1\right)\left[\left\{W_{2}\right\}^{2}+W_{2}\right]}{\left\{\Gamma\left(M_{w}+1\right)\right\}^{-1}\left\{\phi\left(x_{w i}\right)\right\}^{M_{w}+1}} \\
& -\left[\frac{1}{D} \sum_{k_{1}=0}^{r_{1}-1} \sum_{k_{2}=0}^{r_{2}-1} \sum_{k_{3}=0}^{n-s} \sum_{k_{4}=0}^{k_{3}} \prod_{w=1}^{2}(-1)^{k_{3}}\left(\begin{array}{c}
r_{1}-1 \\
k_{1}
\end{array}\right)\left(\begin{array}{c}
r_{2}-1 \\
k_{2}
\end{array}\right)\left(\begin{array}{c}
n-s \\
k_{3}
\end{array}\right)\left(\begin{array}{c}
k_{3} \\
k_{4}
\end{array}\right)\right. \\
& \left.\times \frac{B\left(A_{1}+1, A_{2}+1\right)\left(W_{1}\right)}{\left\{\Gamma\left(M_{w}+1\right)\right\}^{-1}\left\{\phi\left(x_{w i}\right)\right\}^{M_{w}+1}}\right]^{2}
\end{aligned}
$$

\section{Prior elicitation}

The elicitation is method to formulate the prior beliefs regarding some quantities into a probabilistic model. Under Bayesian inference it can be regarded as a technique to specify the values of hyper-parameters in a prior distribution for one or more parameters of the sampling distribution. It is not easy to have an accurate elicitation because in many real life situations, the experts are often not familiar with the concept of probabilities. Even when the expert is familiar with probabilities and their concept, it is by no means straightforward to evaluate a probability value for an event exactly. In such cases, elicitation encourages the expert and the facilitator to consider the meaning of the parameters being elicited. This has two helpful consequences. First, it brings the analysis closer to the application by demanding attention to what is being modeled and what is reasonable to believe about it. Second, it helps to make the posterior distributions, once calculated, into meaningful quantities. Some methods of prior elicitation can be seen from $[3,6,7,13,16,17]$.

We have used the method suggested by [3] for the prior elicitation. This method uses the prior predictive probabilities for elicitation. It compares the prior predictive distribution with expert assessment about this distribution and then to choose the hyper-parameters 
that make the assessment agree closely with the member of the family. The prior predictive distribution can be defined as

$$
g(y)=\int_{0}^{\infty} \int_{0}^{\infty} \int_{0}^{1} g\left(\theta_{1}, \theta_{2}, \pi\right) f\left(y \mid \theta_{1}, \theta_{2}, \pi\right) d \pi d \theta_{1} d \theta_{2} .
$$

where $g\left(\theta_{1}, \theta_{2}, \pi\right)$ is the prior distribution, $f\left(y \mid \theta_{1}, \theta_{2}, \pi\right)$ is mixture density for future observation from Equation (2.3). According to Equation (5.1) the prior predictive distribution under improved gamma prior, presented in Equation (2.1), is

$$
\begin{aligned}
g(y)= & \frac{\operatorname{sech}^{2}(y)}{B\left(a_{3}, b_{3}\right)\{1+\tanh (y)\}} \\
& \times\left[\frac{a_{1} b_{1}^{a_{1}} B\left(a_{3}+1, b_{3}\right)}{\left\{b_{1}-\ln \{0.5(1+\tanh (y))\}\right\}^{a_{1}+1}}+\frac{a_{2} b_{2}^{a_{2}} B\left(a_{3}, b_{3}+1\right)}{\left\{b_{2}-\ln \{0.5(1+\tanh (y))\}\right\}^{a_{2}+1}}\right]
\end{aligned}
$$

As we have to elicit six hyper-parameters so we have to consider six integrals. The set of hyper-parameters with minimum values has been chosen to be the elicited values of the hyper-parameters. By considering the prior predictive distribution in Equation (5.2), we have assumed the experts probabilities to be 0.10 for each integral. We considered the following integrals:

$$
\begin{aligned}
& \int_{-30}^{-20} g(y)=0.10, \\
& \int_{-19.9}^{-10.0} g(y)=0.10, \\
& \int_{-9.9}^{0.0} g(y)=0.10, \\
& \int_{0.1}^{10.0} g(y)=0.10, \\
& \int_{10.1}^{20} g(y)=0.10 \text {, } \\
& \int_{20.1}^{30.0} g(y)=0.10 \text {. }
\end{aligned}
$$

In order to solve these integrals simultaneously, a program has been framed in SAS package using the PROC SYSLIN command and the elicited values of the hyper-parameters have been found to be

$$
\left(a_{1}, b_{1}, a_{2}, b_{2}, a_{3}, b_{3}\right)=(0.8545,1.3184,0.9426,1.6789,0.0216,0.0321) .
$$

Same integrals have been assumed for the elicitation of the hyper-parameters for the remaining priors. However, the above mentioned program has been slightly modified according to the expressions of the respective prior predictive distribution. The prior predictive distribution for improved Jeffreys gamma prior using Equations (2.3) and (3.2) is

$$
\begin{aligned}
g(y)= & \frac{b_{1}^{a_{1}} b_{2}^{a_{2}} \operatorname{sech}^{2}(y)}{\Gamma\left(a_{1}\right) \Gamma\left(a_{2}\right) B\left(a_{3}, b_{3}\right)\{1+\tanh (y)\}} \\
& \times\left[\frac{\Gamma\left(a_{1}\right) \Gamma\left(a_{2}-1\right) B\left(a_{3}+1, b_{3}\right)}{\left\{b_{1}-\ln \{0.5(1+\tanh (y))\}\right\}^{a_{1}} b_{2}^{a_{2}-1}}+\frac{\Gamma\left(a_{1}-1\right) \Gamma\left(a_{2}\right) B\left(a_{3}, b_{3}+1\right)}{b_{1}^{a_{1}-1}\left\{b_{2}-\ln \{0.5(1+\tanh (y))\}\right\}^{a_{2}}}\right] .
\end{aligned}
$$

The elicited values of the hyper-parameters in Equation (5.3) are

$$
\left(a_{1}, b_{1}, a_{2}, b_{2}, a_{3}, b_{3}\right)=(0.7317,1.2183,0.7962,1.4841,0.0196,0.0275) .
$$


Similarly, the prior predictive distribution for improved inverse levy prior using Equations (2.3) and (3.3) is

$$
\begin{aligned}
g(y)= & \frac{\left(b_{4} b_{5}\right)^{\frac{1}{2}} \operatorname{sech}^{2}(y)}{4 B\left(a_{3}, b_{3}\right)\{1+\tanh (y)\}} \\
& \times\left[\left\{\frac{b_{4}}{2}-\ln \left\{\frac{1+\tanh (y)}{2}\right\}\right\}^{-\frac{3}{2}}\left(\frac{b_{5}}{2}\right)^{-\frac{1}{2}} B\left(a_{3}+1, b_{3}\right)\right. \\
& \left.+\left(\frac{b_{4}}{2}\right)^{-\frac{1}{2}}\left\{\frac{b_{5}}{2}-\ln \left\{\frac{1+\tanh (y)}{2}\right\}\right\}^{-\frac{3}{2}} B\left(a_{3}, b_{3}+1\right)\right] .
\end{aligned}
$$

The elicited values of the hyper-parameters in Equation (5.4) are

$$
\left(a_{3}, b_{3}, b_{4}, b_{5}\right)=(0.06315,0.0825,1.0174,0.8526) .
$$

Using Equations (2.3) and (3.4), the prior predictive distribution for improved exponential prior is

$$
\begin{aligned}
g(y) & =\frac{\operatorname{sech}^{2}(y)}{B\left(a_{3}, b_{3}\right)\{1+\tanh (y)\}} \\
& \times\left[\frac{d_{1} B\left(a_{3}+1, b_{3}\right)}{\left\{d_{1}-\ln \{0.5(1+\tanh (y))\}\right\}^{2}}+\frac{d_{2} B\left(a_{3}, b_{3}+1\right)}{\left\{d_{2}-\ln \{0.5(1+\tanh (y))\}\right\}^{2}}\right] .
\end{aligned}
$$

The elicited values of the hyper-parameters in Equation (5.5) are

$$
\left(a_{3}, b_{3}, d_{1}, d_{2}\right)=(0.0316,0.0418,0.0745,0.0587) .
$$

Finally, Equations (2.3) and (3.5), the prior predictive distribution for improved chi square prior is:

$$
\begin{aligned}
g(y) & =\frac{\operatorname{sech}^{2}(y)}{2^{d_{3} / 2} 2^{d_{4} / 2} 2 B\left(a_{3}, b_{3}\right)\{1+\tanh (y)\}} \\
& \times\left[\frac{0.5 d_{3} B\left(a_{3}+1, b_{3}\right)}{\{1.19-\ln \{1+\tanh (y)\}\}^{0.5 d_{3}+1}(0.5)^{0.5 d_{4}}}\right. \\
& \left.+\frac{0.5 d_{4} B\left(a_{3}, b_{3}+1\right)}{(0.5)^{0.5 d_{3}}\{1.19-\ln \{1+\tanh (y)\}\}^{0.5 d_{4}+1}}\right] .
\end{aligned}
$$

The elicited values of the hyper-parameters in Equation (5.6) are

$$
\left(a_{3}, b_{3}, d_{3}, d_{4}\right)=(0.0295,0.0364,1.8169,1.7852) .
$$

\section{Simulation study}

The simulation study is a useful technique to assess the behavior and performance of a proposed system. The results from the study often mimic the real life situations. Therefore, in order to assess and compare the performance of the Bayes estimators with respect to different priors and loss functions and to investigate the impact of sample size on the estimators, a simulation study has been conducted using the parametric space: $\left(\theta_{1}, \theta_{2}, \pi\right) \in(1,2,0.45),(1,2,0.55),(2,4,0.45),(2,4,0.55)$. The probabilistic mixing have been used to draw samples of sizes $\mathrm{n}=20,50,100,150$ and 200 from two components mixture of Burr type VII distribution under 10000 replications. The observations in the respective sample have been assumed to be $20 \%$ censored. In each case, only failures were 
identified to be a member of either first or second sub-population of the mixture. These samples have been drawn by following the listed steps:

(1) Draw samples of size $n$ from the mixture model.

(2) Generate a uniform random no. $\mu$ for each observation.

(3) If $\mu \leq \pi$, the take the observation from first sub-population otherwise from the second sub-population.

(4) Determine the test termination points on left and right, that is, determine the values of $x_{r}$ and $x_{s}$.

(5) The observations which are $<x_{r}$ and $>x_{s}$ have been considered to be censored from each component.

(6) Use the remaining observations from each component for the analysis.

The mathematica software was used to obtain the numerical estimates. The amounts of posterior risks associated with each Bayes estimate have been given in the parenthesis in the tables. The abbreviations used in the tables are: PS: Parametric Set, PS1: $\theta_{1}=1$, $\theta_{2}=2$ and $\pi=0.45 ;$ PS2: $\theta_{1}=1, \theta_{2}=2$ and $\pi=0.55$; PS3: $\theta_{1}=2, \theta_{2}=4$ and $\pi=0.45$; PS4: $\theta_{1}=2, \theta_{2}=4$ and $\pi=0.55$; LL: lower limit; UL: upper limit; NP: Non-informative prior; IGP: Improved Gamma Prior; IJP: Improved Jeffreys Prior; IILP: Improved Inverse Levy Prior; IEP: Improved Exponential Prior; ICSP: Improved Chi Square Prior.

Tables 1-4 represent the amounts of the Bayes estimates and posterior risks for the parameters of the mixture of Burr type VII distribution under doubly censored samples using different priors. From the analysis, it can be assessed that the parameter representing the first component $\left(\theta_{1}\right)$ has been overestimated, while the parameter representing the second component $\left(\theta_{2}\right)$ has been underestimated. The mixing parameter $(\pi)$ has been under estimated for most of the cases. Nonetheless, the Bayes estimates (either under estimated or over estimated) converge to the true value of the parameter as sample size increases. The rate of convergence is bit slower for larger values of the parameters. However, the increase in the values of the mixing parameter leads to relative faster convergence of the estimates (towards the true values of the parameter) of the concerned component. The estimates under the assumption of improved informative priors have faster convergence than those under non-informative prior. On the whole, the rate of convergence is the most under the assumption of improved inverse levy prior. On the other hand, the SLLF provides better convergence than SELF. To be more specific, the estimates under improved inverse levy prior using SLLF are associated with the highest convergence rate. It is interesting to note that the amounts of posterior risks associated with each Bayes estimate decreases by increasing the sample size. This simply indicates that the estimates are consistent. The amounts of posterior risks increase for larger choice of true parametric values; however, the moderate to large sample sizes can face off this problem. In comparison of priors, it has been found that the improve informative priors work better than non-informative prior under both loss functions, as the amounts of posterior risks associated with estimates based on improved informative priors are smaller than those under non-informative prior. The minimum amounts of posterior risks have been observed for the estimates on the basis of improved inverse levy prior. In order of efficiency (amounts of posterior risks), the performance of the priors can be ranked as: improved inverse levy prior (1), improved Jeffreys gamma prior (2), improved chi square prior (3), improved gamma prior (4), improved exponential prior (5), non-informative prior (6) with some exceptions. In contrast, the estimates under SLLF are superior to those under SELF. Hence, for the point estimation of the parameters of the mixed Burr type VII model, the use of inverse levy prior under SLLF may be preferred. 
Table 1. The Bayes estimates and posterior risks based on NP, IGP and IJP under SLLF using PS1, PS2, PS3 and PS4, respectively.

\begin{tabular}{|c|c|c|c|c|c|c|c|c|c|}
\hline \multirow[t]{2}{*}{$\mathrm{n}$} & \multicolumn{3}{|c|}{ Non-informative Prior } & \multicolumn{3}{|c|}{ Improved Gamma Prior } & \multicolumn{3}{|c|}{$\begin{array}{c}\text { Improved Jeffreys Gamma } \\
\text { Prior }\end{array}$} \\
\hline & $\hat{\theta}_{1}$ & $\hat{\theta}_{2}$ & $\hat{\pi}$ & $\hat{\theta}_{1}$ & $\hat{\theta}_{2}$ & $\hat{\pi}$ & $\hat{\theta}_{1}$ & $\hat{\theta}_{2}$ & $\hat{\pi}$ \\
\hline 20 & $\begin{array}{c}1.36678 \\
(0.00627)\end{array}$ & $\begin{array}{c}1.65857 \\
(0.00706)\end{array}$ & $\begin{array}{c}0.43948 \\
(0.00490)\end{array}$ & $\begin{array}{c}1.36102 \\
(0.00524)\end{array}$ & $\begin{array}{c}1.64905 \\
(0.00664)\end{array}$ & $\begin{array}{c}0.43769 \\
(0.00449)\end{array}$ & $\begin{array}{c}1.33324 \\
(0.00501)\end{array}$ & $\begin{array}{c}1.64487 \\
(0.00602)\end{array}$ & $\begin{array}{c}0.43591 \\
(0.00578)\end{array}$ \\
\hline 50 & $\begin{array}{c}1.17319 \\
(0.00570)\end{array}$ & $\begin{array}{c}1.74046 \\
(0.00643)\end{array}$ & $\begin{array}{c}0.44222 \\
(0.00434)\end{array}$ & $\begin{array}{c}1.16824 \\
(0.00477)\end{array}$ & $\begin{array}{c}1.73046 \\
(0.00604)\end{array}$ & $\begin{array}{c}0.44042 \\
(0.00398)\end{array}$ & $\begin{array}{c}1.14439 \\
(0.00449)\end{array}$ & $\begin{array}{c}1.72608 \\
(0.00548)\end{array}$ & $\begin{array}{c}0.43862 \\
(0.00511)\end{array}$ \\
\hline 100 & $\begin{array}{c}1.08085 \\
(0.00513)\end{array}$ & $\begin{array}{c}1.80232 \\
(0.00549)\end{array}$ & $\begin{array}{c}0.44428 \\
(0.00371)\end{array}$ & $\begin{array}{c}1.07630 \\
(0.00428)\end{array}$ & $\begin{array}{c}1.79196 \\
(0.00517)\end{array}$ & \begin{tabular}{|c|}
0.44247 \\
$(0.00340)$
\end{tabular} & $\begin{array}{c}1.05433 \\
(0.00403)\end{array}$ & $\begin{array}{c}1.78742 \\
(0.00469)\end{array}$ & $\begin{array}{c}0.44066 \\
(0.00438)\end{array}$ \\
\hline 150 & $\begin{array}{c}1.05023 \\
(0.00419)\end{array}$ & $\begin{array}{c}1.89440 \\
(0.00439)\end{array}$ & \begin{tabular}{|c|}
0.44644 \\
$(0.00296)$
\end{tabular} & $\begin{array}{c}1.04579 \\
(0.00349)\end{array}$ & $\begin{array}{c}1.88351 \\
(0.00412) \\
\end{array}$ & $\begin{array}{c}0.44462 \\
(0.00271)\end{array}$ & $\begin{array}{c}1.02445 \\
(0.00329)\end{array}$ & $\begin{array}{c}1.87875 \\
(0.00374)\end{array}$ & $\begin{array}{c}0.44280 \\
(0.00349)\end{array}$ \\
\hline 200 & $\begin{array}{c}1.01715 \\
(0.00315)\end{array}$ & $\begin{array}{c}1.94293 \\
(0.00342)\end{array}$ & $\begin{array}{c}0.45011 \\
(0.00231)\end{array}$ & $\begin{array}{c}1.01286 \\
(0.00263)\end{array}$ & $\begin{array}{c}1.93178 \\
(0.00322)\end{array}$ & $\begin{array}{c}0.44827 \\
(0.00212)\end{array}$ & $\begin{array}{c}1.01699 \\
(0.00247)\end{array}$ & $\begin{array}{c}1.92688 \\
(0.00291)\end{array}$ & $\begin{array}{c}0.44644 \\
(0.00272)\end{array}$ \\
\hline 20 & $\begin{array}{c}1.34688 \\
(0.00568)\end{array}$ & $\begin{array}{c}1.63262 \\
(0.00738)\end{array}$ & $\begin{array}{c}0.52737 \\
(0.00562)\end{array}$ & $\begin{array}{c}1.34119 \\
(0.00474)\end{array}$ & $\begin{array}{c}1.62324 \\
(0.00694)\end{array}$ & $\begin{array}{c}0.52523 \\
(0.00514)\end{array}$ & $\begin{array}{c}1.31382 \\
(0.00454)\end{array}$ & $\begin{array}{c}1.61914 \\
(0.00629)\end{array}$ & $\begin{array}{c}0.52308 \\
(0.00662)\end{array}$ \\
\hline 50 & $\begin{array}{c}1.15610 \\
(0.00517)\end{array}$ & $\begin{array}{c}1.71323 \\
(0.00672)\end{array}$ & \begin{tabular}{|c|}
0.53066 \\
$(0.00497)$
\end{tabular} & $\begin{array}{c}1.15122 \\
(0.00432) \\
\end{array}$ & $\begin{array}{c}1.70339 \\
(0.00632) \\
\end{array}$ & $\begin{array}{c}0.52850 \\
(0.00455) \\
\end{array}$ & $\begin{array}{c}1.12773 \\
(0.00407) \\
\end{array}$ & $\begin{array}{c}1.69908 \\
(0.00573)\end{array}$ & $\begin{array}{c}0.52634 \\
(0.00586)\end{array}$ \\
\hline 100 & $\begin{array}{c}1.06511 \\
(0.00464)\end{array}$ & $\begin{array}{c}1.77411 \\
(0.00575)\end{array}$ & \begin{tabular}{|c|}
0.53313 \\
$(0.00425)$
\end{tabular} & $\begin{array}{c}1.06061 \\
(0.00388)\end{array}$ & \begin{tabular}{|c|}
1.76393 \\
$(0.00541)$ \\
\end{tabular} & $\begin{array}{c}0.53096 \\
(0.00390) \\
\end{array}$ & $\begin{array}{c}1.03897 \\
(0.00366)\end{array}$ & $\begin{array}{c}1.75946 \\
(0.00491)\end{array}$ & $\begin{array}{c}0.52880 \\
(0.00502) \\
\end{array}$ \\
\hline 150 & $\begin{array}{c}1.03494 \\
(0.00379)\end{array}$ & $\begin{array}{c}1.86475 \\
(0.00457)\end{array}$ & $\begin{array}{c}0.53572 \\
(0.00339)\end{array}$ & $\begin{array}{c}1.03056 \\
(0.00316)\end{array}$ & $\begin{array}{c}1.85404 \\
(0.00431)\end{array}$ & $\begin{array}{c}0.53354 \\
(0.00310)\end{array}$ & $\begin{array}{c}1.00954 \\
(0.00298)\end{array}$ & $\begin{array}{c}1.84935 \\
(0.00391)\end{array}$ & $\begin{array}{c}0.53136 \\
(0.00399)\end{array}$ \\
\hline 200 & $\begin{array}{c}1.02584 \\
(0.00285)\end{array}$ & $\begin{array}{c}1.91253 \\
(0.00357)\end{array}$ & $\begin{array}{c}0.54013 \\
(0.00265)\end{array}$ & $\begin{array}{c}1.02150 \\
(0.00238)\end{array}$ & $\begin{array}{c}1.90155 \\
(0.00336)\end{array}$ & \begin{tabular}{|c|}
0.53793 \\
$(0.00242)$
\end{tabular} & $\begin{array}{c}1.02568 \\
(0.00224)\end{array}$ & $\begin{array}{c}1.89673 \\
(0.00305)\end{array}$ & $\begin{array}{c}0.53572 \\
(0.00312)\end{array}$ \\
\hline 20 & $\begin{array}{c}2.53951 \\
(0.00759)\end{array}$ & $\begin{array}{c}3.32142 \\
(0.00870)\end{array}$ & \begin{tabular}{|c|}
0.42825 \\
$(0.00805)$ \\
\end{tabular} & $\begin{array}{c}2.67466 \\
(0.00957)\end{array}$ & $\begin{array}{c}3.47754 \\
(0.01163) \\
\end{array}$ & \begin{tabular}{|c|}
0.43408 \\
$(0.00787)$ \\
\end{tabular} & $\begin{array}{c}2.50496 \\
(0.00576) \\
\end{array}$ & $\begin{array}{c}3.28757 \\
(0.00740) \\
\end{array}$ & \begin{tabular}{|c|}
0.43991 \\
$(0.00576)$
\end{tabular} \\
\hline 50 & $\begin{array}{c}2.17980 \\
(0.00690)\end{array}$ & $\begin{array}{c}3.48540 \\
(0.00792)\end{array}$ & $\begin{array}{c}0.43092 \\
(0.00713)\end{array}$ & $\begin{array}{c}2.29581 \\
(0.00871)\end{array}$ & $\begin{array}{c}3.64923 \\
(0.01059)\end{array}$ & \begin{tabular}{|c|}
0.43678 \\
$(0.00696)$
\end{tabular} & $\begin{array}{c}2.15015 \\
(0.00524)\end{array}$ & $\begin{array}{c}3.44987 \\
(0.00674)\end{array}$ & $\begin{array}{c}0.44264 \\
(0.00511)\end{array}$ \\
\hline 100 & $\begin{array}{c}2.12875 \\
(0.00620)\end{array}$ & $\begin{array}{c}3.60927 \\
(0.00678)\end{array}$ & $\begin{array}{c}0.43293 \\
(0.00611)\end{array}$ & $\begin{array}{c}2.24203 \\
(0.00783)\end{array}$ & $\begin{array}{c}3.77893 \\
(0.00906)\end{array}$ & \begin{tabular}{|c|}
0.43881 \\
$(0.00596)$
\end{tabular} & $\begin{array}{c}2.09978 \\
(0.00471)\end{array}$ & $\begin{array}{c}3.57249 \\
(0.00577)\end{array}$ & $\begin{array}{c}0.44470 \\
(0.00437)\end{array}$ \\
\hline 150 & $\begin{array}{c}2.08642 \\
(0.00506)\end{array}$ & \begin{tabular}{|c|}
3.79367 \\
$(0.00541)$ \\
\end{tabular} & $\begin{array}{c}0.43503 \\
(0.00486)\end{array}$ & $\begin{array}{c}2.19746 \\
(0.00639)\end{array}$ & $\begin{array}{c}3.97199 \\
(0.00722) \\
\end{array}$ & \begin{tabular}{|c|}
0.44095 \\
$(0.00475)$ \\
\end{tabular} & $\begin{array}{c}2.05803 \\
(0.00384)\end{array}$ & $\begin{array}{c}3.75501 \\
(0.00459)\end{array}$ & $\begin{array}{c}0.44686 \\
(0.00348)\end{array}$ \\
\hline 200 & $\begin{array}{c}2.03753 \\
(0.00381)\end{array}$ & $\begin{array}{c}3.89086 \\
(0.00422)\end{array}$ & \begin{tabular}{|c|}
0.43860 \\
$(0.00380)$
\end{tabular} & $\begin{array}{c}2.14597 \\
(0.00480)\end{array}$ & $\begin{array}{c}4.07375 \\
(0.00564) \\
\end{array}$ & $\begin{array}{c}0.44457 \\
(0.00371)\end{array}$ & $\begin{array}{c}2.02991 \\
(0.00289) \\
\end{array}$ & $\begin{array}{c}3.85121 \\
(0.00359) \\
\end{array}$ & $\begin{array}{c}0.45054 \\
(0.00272)\end{array}$ \\
\hline 20 & $\begin{array}{c}2.50253 \\
(0.00687)\end{array}$ & $\begin{array}{c}3.26946 \\
(0.00910)\end{array}$ & $\begin{array}{c}0.51390 \\
(0.00923)\end{array}$ & $\begin{array}{c}2.63571 \\
(0.00867)\end{array}$ & $\begin{array}{c}3.42313 \\
(0.01215) \\
\end{array}$ & $\begin{array}{c}0.52089 \\
(0.00901)\end{array}$ & $\begin{array}{c}2.46848 \\
(0.00522)\end{array}$ & $\begin{array}{c}3.23614 \\
(0.00774)\end{array}$ & $\begin{array}{c}0.52789 \\
(0.00660)\end{array}$ \\
\hline 50 & $\begin{array}{c}2.14806 \\
(0.00625)\end{array}$ & $\begin{array}{c}3.43086 \\
(0.00830)\end{array}$ & \begin{tabular}{|c|}
0.51711 \\
$(0.00817)$
\end{tabular} & $\begin{array}{c}2.26237 \\
(0.00789)\end{array}$ & $\begin{array}{c}3.59213 \\
(0.01107)\end{array}$ & $\begin{array}{c}0.52414 \\
(0.00798)\end{array}$ & $\begin{array}{c}2.11883 \\
(0.00475)\end{array}$ & $\begin{array}{c}3.39591 \\
(0.00705)\end{array}$ & $\begin{array}{c}0.53118 \\
(0.00585)\end{array}$ \\
\hline 100 & $\begin{array}{c}2.09774 \\
(0.00562)\end{array}$ & $\begin{array}{c}3.55280 \\
(0.00709)\end{array}$ & $\begin{array}{c}0.51950 \\
(0.00700) \\
\end{array}$ & $\begin{array}{c}2.20938 \\
(0.00709)\end{array}$ & $\begin{array}{c}3.71981 \\
(0.00948) \\
\end{array}$ & $\begin{array}{c}0.52657 \\
(0.00683) \\
\end{array}$ & $\begin{array}{c}2.06920 \\
(0.00427) \\
\end{array}$ & $\begin{array}{c}3.51659 \\
(0.00603)\end{array}$ & $\begin{array}{c}0.53364 \\
(0.00501) \\
\end{array}$ \\
\hline 150 & $\begin{array}{c}2.05603 \\
(0.00459)\end{array}$ & $\begin{array}{c}3.73432 \\
(0.00564)\end{array}$ & \begin{tabular}{|c|}
0.52204 \\
$(0.00557)$ \\
\end{tabular} & $\begin{array}{c}2.16545 \\
(0.00578)\end{array}$ & $\begin{array}{c}3.90985 \\
(0.00755) \\
\end{array}$ & \begin{tabular}{|c|}
0.52914 \\
$(0.00544)$ \\
\end{tabular} & $\begin{array}{c}2.02806 \\
(0.00348) \\
\end{array}$ & $\begin{array}{c}3.69626 \\
(0.00480)\end{array}$ & \begin{tabular}{|c|}
0.53624 \\
$(0.00399)$ \\
\end{tabular} \\
\hline 200 & $\begin{array}{c}2.05495 \\
(0.00344)\end{array}$ & $\begin{array}{c}3.83000 \\
(0.00442)\end{array}$ & $\begin{array}{c}0.52632 \\
(0.00435)\end{array}$ & $\begin{array}{c}2.16430 \\
(0.00435)\end{array}$ & $\begin{array}{c}4.01002 \\
(0.00589)\end{array}$ & $\begin{array}{c}0.53348 \\
(0.00425)\end{array}$ & $\begin{array}{c}2.04725 \\
(0.00262)\end{array}$ & $\begin{array}{c}3.79096 \\
(0.00375)\end{array}$ & $\begin{array}{c}0.54064 \\
(0.00311)\end{array}$ \\
\hline
\end{tabular}


Table 2. The Bayes estimates and posterior risks based on NP, IGP and IJP under SELF using PS1, PS2, PS3 and PS4 respectively .

\begin{tabular}{|c|c|c|c|c|c|c|c|c|c|}
\hline \multirow[t]{2}{*}{$\mathrm{n}$} & \multicolumn{3}{|c|}{ Non-informative Prior } & \multicolumn{3}{|c|}{ Improved Gamma Prior } & \multicolumn{3}{|c|}{$\begin{array}{c}\text { Improved Jeffreys Gamma } \\
\text { Prior }\end{array}$} \\
\hline & $\hat{\theta}_{1}$ & $\hat{\theta}_{2}$ & $\hat{\pi}$ & $\hat{\theta}_{1}$ & $\hat{\theta}_{2}$ & $\hat{\pi}$ & $\hat{\theta}_{1}$ & $\hat{\theta}_{2}$ & $\hat{\pi}$ \\
\hline \multirow[t]{2}{*}{20} & 1.39412 & 1.62540 & 0.43377 & 1.38824 & 1.61607 & 0.43200 & 1.37842 & 1.61197 & 0.43024 \\
\hline & $(0.01037)$ & $(0.01230)$ & $(0.00803)$ & $(0.00919)$ & $(0.01223)$ & $(0.00780)$ & $(0.00853)$ & $(0.01110)$ & $(0.01003)$ \\
\hline \multirow{2}{*}{50} & 1.19665 & 1.70565 & 0.43647 & 1.19160 & 1.69586 & 0.43470 & 1.18317 & 1.69156 & 0.43292 \\
\hline & $(0.00944)$ & $(0.01121)$ & $(0.00710)$ & $(0.00836)$ & $(0.01114)$ & $(0.00691)$ & $(0.00777)$ & $(0.01011)$ & \\
\hline \multirow{2}{*}{100} & 1.10247 & 1.76627 & 0.43850 & 1.09782 & \begin{tabular}{|l|}
1.75612 \\
\end{tabular} & 0.43672 & 1.09005 & 1.75167 & 0.43493 \\
\hline & $(0.00849)$ & $(0.00957)$ & $(0.00608)$ & $(0.00752)$ & $(0.00954)$ & $(0.00591)$ & $(0.00698)$ & $(0.00866)$ & $(0.00761)$ \\
\hline \multirow{2}{*}{150} & 1.07123 & 1.85651 & 0.44064 & 1.06671 & 1.84584 & 0.43884 & 1.05917 & 1.84117 & 0.43705 \\
\hline & $(0.00693)$ & $(0.00765)$ & $(0.00484)$ & $(0.00613)$ & $(0.00760)$ & $(0.00471)$ & $(0.00569)$ & $(0.00689)$ & $(0.00606)$ \\
\hline \multirow{2}{*}{200} & \begin{tabular}{|l|}
1.03749 \\
\end{tabular} & 1.90407 & 0.44426 & 1.03312 & 1.89314 & 0.44244 & \begin{tabular}{|c|}
1.02580 \\
\end{tabular} & 1.88834 & 0.44064 \\
\hline & $(0.00521)$ & $(0.00596)$ & $(0.00378)$ & $(0.00461)$ & $(0.00593)$ & $(0.00368)$ & $(0.00428)$ & $(0.00538)$ & $(0.00473)$ \\
\hline \multirow{2}{*}{20} & 1.37381 & 1.59997 & 0.52052 & 1.36801 & 1.59078 & 0.51840 & 1.35834 & 1.58675 & \\
\hline & $(0.00940)$ & $(0.01287)$ & (0.00920) & $(0.00832)$ & $(0.01279)$ & $(0.00894)$ & $(0.00773)$ & $(0.01161)$ & 150) \\
\hline \multirow{2}{*}{50} & 1.17922 & 1.67896 & 0.52377 & 1.17424 & 1.66932 & 0.52163 & 1.16594 & 1.66509 & 0.51950 \\
\hline & $(0.00856)$ & $(0.01171)$ & $(0.00815)$ & $(0.00757)$ & $(0.01165)$ & $(0.00791)$ & $(0.00703)$ & $(0.01057)$ & $(0.01018)$ \\
\hline \multirow{2}{*}{100} & 1.08641 & 1.73863 & 0.52620 & 1.08182 & 1.72865 & 0.52405 & 1.07418 & 1.72427 & 2192 \\
\hline & $(0.00768)$ & $(0.01002)$ & $(0.00697)$ & $(0.00681)$ & $(0.00997)$ & $(0.00678)$ & $(0.00632)$ & $(0.00905)$ & 871) \\
\hline \multirow{2}{*}{150} & 1.05564 & 1.82746 & 0.52876 & 1.05117 & 1.81696 & 0.52661 & 1.04375 & 1.81236 & 0.52445 \\
\hline & $(0.00627)$ & $(0.00797)$ & $(0.00555)$ & $(0.00555)$ & $(0.00794)$ & $(0.00539)$ & $(0.00516)$ & $(0.00720)$ & $(0.0$ \\
\hline \multirow[t]{2}{*}{200} & 1.04635 & 1.87428 & 0.53311 & 1.04193 & 1.86352 & \begin{tabular}{|l|}
0.53093 \\
\end{tabular} & 1.03457 & 1.85880 & 0.52876 \\
\hline & $(0.00471)$ & $(0.00623)$ & $(0.00433)$ & $(0.00417)$ & $(0.00620)$ & $(0.00421)$ & $(0.00388)$ & $(0.00562)$ & $(0.00542)$ \\
\hline \multirow{2}{*}{20} & 2.59030 & 3.25499 & 0.42268 & 2.72816 & 3.40799 & 0.42843 & 2.55506 & 3.22182 & 3419 \\
\hline & $(0.01255)$ & $(0.01518)$ & (0.01319) & $(0.016$ & $(0.02143)$ & $(0.01367)$ & $(0.01$ & $(0.01365)$ & $002)$ \\
\hline \multirow{2}{*}{50} & 2.22340 & 3.41569 & 0.42531 & 2.34173 & 3.57624 & 0.43111 & 2.19315 & 3.38088 & \begin{tabular}{|l|}
0.43689 \\
\end{tabular} \\
\hline & $(0.01142)$ & $(0.01381)$ & (0.01168) & $(0.01529)$ & $(0.01952)$ & $(0.01210)$ & $(0.00920)$ & $(0.01243)$ & $(0.00887)$ \\
\hline \multirow{2}{*}{100} & 2.17132 & 3.53709 & 0.42730 & 2.28687 & 3.70335 & 0.43311 & 2.14178 & 3.50104 & 0.43892 \\
\hline & $(0.01026)$ & $(0.01183)$ & $(0.01000)$ & $(0.01374)$ & $(0.01671)$ & $(0.01036)$ & $(0.00827)$ & $(0.01064)$ & $(0.00760)$ \\
\hline \multirow{2}{*}{150} & 2.12815 & 3.71779 & 0.42937 & 2.24141 & 3.89255 & 0.43521 & 2.09919 & 3.67991 & 0.44106 \\
\hline & $(0.00838)$ & $(0.00943)$ & $(0.00796)$ & $(0.01121)$ & $(0.01331)$ & $(0.00825)$ & $(0.00674)$ & $(0.00847)$ & $(0.00605)$ \\
\hline \multirow{2}{*}{200} & 2.07828 & 3.81305 & 0.43290 & 2.18889 & 3.99228 & 0.43879 & 2.07051 & 3.77419 & 0.44468 \\
\hline & $(0.00630)$ & $(0.00735)$ & $(0.00622)$ & $(0.00843)$ & $(0.01039)$ & $(0.00644)$ & $(0.00507)$ & $(0.00662)$ & $(0.00472)$ \\
\hline \multirow{2}{*}{20} & 2.55258 & 3.20407 & 0.50722 & 2.68842 & 3.35467 & 0.51412 & 2.51785 & 3.17141 & 0.52102 \\
\hline & $(0.01136)$ & $(0.01586)$ & $(0.01511)$ & $(0.01521)$ & $(0.02241)$ & $(0.01566)$ & $(0.00915)$ & $(0.01427)$ & $(0.01148)$ \\
\hline \multirow{2}{*}{50} & 2.19102 & 3.36225 & 0.51039 & 2.30762 & 3.52029 & 0.51733 & 2.16121 & 3.32799 & 0.52427 \\
\hline & $(0.01034)$ & $(0.01447)$ & $(0.01338)$ & $(0.01384)$ & $(0.02041)$ & $(0.01387)$ & $(0.00833)$ & $(0.01299)$ & $(0.01016)$ \\
\hline \multirow{2}{*}{100} & 2.13969 & 3.48175 & 0.51275 & 2.25356 & 3.64541 & 0.51973 & 2.11058 & 3.44626 & 0.52671 \\
\hline & $(0.00929)$ & $(0.01236)$ & $(0.01146)$ & $(0.01244)$ & $(0.01747)$ & $(0.01187)$ & $(0.00749)$ & $(0.01113)$ & $(0.00870)$ \\
\hline \multirow{2}{*}{150} & 2.09715 & 3.65963 & 0.51525 & 2.20876 & 3.83165 & 0.52226 & 2.06862 & 3.62233 & 0.52927 \\
\hline & $(0.00759)$ & $(0.00984)$ & $(0.00912)$ & $(0.01015)$ & $(0.01391)$ & $(0.00945)$ & $(0.00611)$ & $(0.00886)$ & $(0.00693)$ \\
\hline 200 & 2.09604 & 3.75340 & 0.51948 & 2.20758 & 3.92982 & 0.52655 & 2.08819 & 3.71514 & 0.53361 \\
\hline & $(0.00560)$ & (0.001/1) & $(0.00712)$ & $(0.00763)$ & $(0.01086)$ & $(0.00738)$ & $(0.00459)$ & $(0.00691)$ & $(0.00541)$ \\
\hline
\end{tabular}


Table 3. The Bayes estimates and posterior risks based on IILP, IEP and ICSP under SLLF using PS1, PS2, PS3 and PS4 respectively.

\begin{tabular}{|c|c|c|c|c|c|c|c|c|c|}
\hline \multirow[t]{2}{*}{$\mathrm{n}$} & \multicolumn{3}{|c|}{ Non-informative Prior } & \multicolumn{3}{|c|}{ Improved Gamma Prior } & \multicolumn{3}{|c|}{ Improved Jeffreys Gamma } \\
\hline & $\hat{\theta}_{1}$ & $\hat{\theta}_{2}$ & $\hat{\pi}$ & $\hat{\theta}_{1}$ & $\hat{\theta}_{2}$ & $\hat{\pi}$ & $\hat{\theta}_{1}$ & $\hat{\theta}_{2}$ & $\hat{\pi}$ \\
\hline \multirow{2}{*}{20} & 1.35139 & 1.73396 & 0.43833 & 1.34733 & 1.63605 & 0.43655 & 1.34433 & 1.63191 & 0.43477 \\
\hline & $(0.00486)$ & $(0.00563)$ & $(0.00391)$ & $(0.00591)$ & $(0.00681)$ & $(0.00490)$ & $(0.00526)$ & $(0.00668)$ & $(0.00527)$ \\
\hline \multirow{2}{*}{50} & 1.15997 & \begin{tabular}{|l|}
1.81957 \\
\end{tabular} & 0.44106 & 1.15649 & 1.71682 & 0.43927 & 1.15391 & \begin{tabular}{|l|}
1.71247 \\
\end{tabular} & \\
\hline & $(0.00443)$ & $(0.00500)$ & $(0.00343)$ & $(0.00529)$ & $(0.00604)$ & $(0.00429)$ & $(0.00471)$ & $(0.00592)$ & $(0.00461)$ \\
\hline \multirow{2}{*}{100} & 1.06868 & 1.88424 & 0.44311 & 1.06547 & 1.77783 & 0.44131 & 1.06309 & 1.77333 & 3951 \\
\hline & $(0.00398)$ & $(0.00427)$ & $(0.00293)$ & $(0.00476)$ & $(0.00517)$ & $(0.00367)$ & $(0.00423)$ & $(0.00507)$ & $(0.00395)$ \\
\hline \multirow{2}{*}{150} & 1.03840 & 1.90301 & 0.44527 & 1.03528 & 1.79554 & 0.44346 & 1.03298 & 1.79100 & \\
\hline & $(0.00324)$ & $(0.00340)$ & $(0.00233)$ & $(0.00388)$ & $(0.00412)$ & $(0.00292)$ & $(0.00345)$ & $(0.00404)$ & $(0.00314)$ \\
\hline \multirow{2}{*}{200} & 1.00569 & 1.95177 & 0.44894 & 1.02774 & 1.84155 & 0.44710 & 1.02545 & 1.83689 & 0.44528 \\
\hline & $(0.00244)$ & $(0.00265)$ & $(0.00182)$ & $(0.00292)$ & $(0.00322)$ & $(0.00228)$ & $(0.00260)$ & $(0.00315)$ & $(0.00246)$ \\
\hline \multirow{2}{*}{20} & 1.33171 & 1.70684 & 0.52600 & 1.32770 & 1.61045 & 0.52386 & 1.32474 & 1.60637 & 0.52172 \\
\hline & $(0.00440)$ & $(0.00588)$ & $(0.00448)$ & $(0.00535)$ & $(0.00712)$ & $(0.00561)$ & $(0.00476)$ & $(0.00698)$ & 604) \\
\hline \multirow{2}{*}{50} & 1.14308 & 1.791 & 0.52928 & 1.13965 & 1.68996 & 0.52 & 1.13710 & 1.68568 & \\
\hline & $(0.00401)$ & $(0.005$ & $(0.00392)$ & $(0.004$ & $(0.00632)$ & $(0.00$ & $(0.00427)$ & $(0.00619)$ & \\
\hline \multirow{2}{*}{100} & 1.05312 & 1.85476 & 0.53174 & 1.04995 & 1.75002 & 0.52958 & 1.04761 & 1.74559 & 741 \\
\hline & $(0.00360)$ & $(0.00447)$ & $(0.00336)$ & $(0.00431)$ & $(0.00541)$ & $(0.00420)$ & $(0.00383)$ & $(0.00530)$ & $(0.00452)$ \\
\hline \multirow[t]{2}{*}{150} & 1.02328 & 1.87323 & 0.53433 & 1.02020 & 1.76746 & 0.53215 & \begin{tabular}{|l|}
1.01793 \\
\end{tabular} & \begin{tabular}{|c|}
1.76298 \\
\end{tabular} & 998 \\
\hline & $(0.00294)$ & $(0.00355)$ & $(0.00268)$ & $(0.00351)$ & $(0.00431)$ & $(0.00335)$ & $(0.00313)$ & $(0.00422)$ & 360) \\
\hline \multirow{2}{*}{200} & 1.01428 & 1.92123 & 0.53872 & 1.03652 & 1.81274 & 0.53652 & 1.03420 & 1.80814 & 0.53433 \\
\hline & $(0.00221)$ & $(0.00277)$ & $(0.00209)$ & $(0.00264)$ & $(0.00336)$ & $(0.00261)$ & $(0.00235)$ & $(0.00329)$ & 281) \\
\hline \multirow{2}{*}{20} & 2.47719 & 3.47239 & 0.42714 & 2.64776 & 3.45012 & 0.43295 & 2.49187 & 3.26165 & 876 \\
\hline & $(0.00606)$ & $(0.00695)$ & $(0.00643)$ & $(0.01079)$ & $(0.01194)$ & $(0.00858)$ & $(0.00623)$ & $(0.00821)$ & $(0.00526)$ \\
\hline \multirow{2}{*}{50} & 2.12630 & 3.64383 & 0.42980 & 2.27272 & 3.62046 & 0.43565 & 2.13891 & 3.42268 & 0.44149 \\
\hline & $(0.00543)$ & $(0.00617)$ & $(0.00563)$ & $(0.00968)$ & $(0.01059)$ & $(0.00751)$ & $(0.00558)$ & $(0.00728$ & 460) \\
\hline \multirow{2}{*}{100} & 2.11568 & 3.77333 & 0.43180 & 2.26137 & 3.74914 & 0.43767 & 2.12822 & 3.54432 & 0.44355 \\
\hline & $(0.00488)$ & $(0.00529)$ & $(0.00482)$ & $(0.00870)$ & $(0.00906)$ & $(0.00643)$ & $(0.00502)$ & $(0.00623)$ & $(0.00394)$ \\
\hline \multirow{2}{*}{150} & 2.05573 & 3.81092 & 0.43390 & 2.19729 & 3.78648 & 0.43980 & 2.06792 & 3.57963 & 0.44571 \\
\hline & & $(0.00420)$ & $(0.00383)$ & $(0.00709)$ & $(0.00722)$ & $(0.00512)$ & $(0.00409)$ & $(0.00496)$ & 314) \\
\hline \multirow{2}{*}{200} & 2.04076 & 3.90855 & 0.43746 & 2.18128 & 3.88349 & 0.44341 & 2.05285 & 3.67134 & 0.44936 \\
\hline & $(0.00299)$ & $(0.00328)$ & $(0.00299)$ & $(0.00533)$ & $(0.00563)$ & $(0.00400)$ & $(0.00308)$ & $(0.00387)$ & $(0.00245)$ \\
\hline \multirow{2}{*}{20} & 2.44111 & 3.41807 & 0.51256 & 2.60920 & 3.39615 & 0.51953 & 2.45557 & 3.21062 & 0.52651 \\
\hline & $(0.00548)$ & $(0.00728)$ & $(0.00737)$ & $(0.00977)$ & $(0.01248)$ & $(0.00984)$ & $(0.00564)$ & $(0.00858)$ & $(0.00603)$ \\
\hline \multirow{2}{*}{50} & 2.09534 & 3.58682 & 0.51576 & 2.23963 & 3.56382 & 0.52278 & 2.10775 & 3.36913 & 0.52979 \\
\hline & $(0.00492)$ & $(0.00644)$ & $(0.00645)$ & $(0.00876)$ & $(0.01107)$ & $(0.00860)$ & $(0.00505)$ & $(0.00761)$ & $(0.00527)$ \\
\hline \multirow[t]{2}{*}{100} & 2.08486 & 3.71430 & 0.51816 & 2.22842 & 3.69048 & 0.52520 & 2.09721 & 3.48888 & 0.53225 \\
\hline & $(0.00442)$ & $(0.00553)$ & $(0.00552)$ & $(0.00787)$ & $(0.00947)$ & $(0.00737)$ & $(0.00454)$ & $(0.00652)$ & $(0.00452)$ \\
\hline \multirow[t]{2}{*}{150} & 2.02580 & 3.75129 & 0.52068 & 2.16529 & 3.72724 & 0.52776 & 2.03780 & 3.52362 & 0.53485 \\
\hline & $(0.00360)$ & $(0.00439)$ & $(0.00440)$ & $(0.00642)$ & $(0.00754)$ & $(0.00587)$ & $(0.00370)$ & $(0.00519)$ & $(0.00360)$ \\
\hline 200 & 2.05819 & 3.84741 & 0.52496 & 2.19992 & 3.82274 & 0.53210 & 2.07038 & 3.61391 & 0.53923 \\
\hline 200 & $(0.00271)$ & $(0.00343)$ & $(0.00343)$ & $(0.00483)$ & $(0.00589)$ & $(0.00458)$ & $(0.00279)$ & $(0.00405)$ & $(0.00281)$ \\
\hline
\end{tabular}


Table 4. The Bayes estimates and posterior risks based on IILP, IEP and ICSP under SELF using PS1, PS2, PS3 and PS4 respectively.

\begin{tabular}{|c|c|c|c|c|c|c|c|c|c|}
\hline \multirow[t]{2}{*}{$\mathrm{n}$} & \multicolumn{3}{|c|}{ Non-informative Prior } & \multicolumn{3}{|c|}{ Improved Gamma Prior } & \multicolumn{3}{|c|}{$\begin{array}{c}\text { Improved Jeffreys Gamma } \\
\text { Prior }\end{array}$} \\
\hline & $\hat{\theta}_{1}$ & $\hat{\theta}_{2}$ & $\hat{\pi}$ & $\hat{\theta}_{1}$ & $\hat{\theta}_{2}$ & $\hat{\pi}$ & $\hat{\theta}_{1}$ & $\hat{\theta}_{2}$ & $\hat{\pi}$ \\
\hline \multirow{2}{*}{20} & & & 043263 & & 160222 & & 137121 & 1.59927 & \\
\hline & & & & & & & & $(0.01232)$ & \\
\hline \multirow{2}{*}{50} & & & & & & & & 1.67822 & \\
\hline & & $(0.00$ & $(0.00561)$ & $(0.009$ & & & & $(0.01092)$ & \\
\hline \multirow{2}{*}{100} & & & & & & & & 1.73787 & \\
\hline & & & & $(0$ & 0954) & & $(0.0$ & $(0.00935)$ & \\
\hline \multirow{2}{*}{150} & & & & & & & & 1.75518 & \\
\hline & $(0.00544)$ & & $(0.00382)$ & $(0.00$ & $759)$ & & $(0.0$ & $(0.00744)$ & \\
\hline \multirow{2}{*}{200} & & & & & & & & 1.80015 & \\
\hline & $(0.00409)$ & & 99) & & & & & $(0.00581)$ & 27) \\
\hline \multirow[t]{2}{*}{20} & 1.34009 & & & & & & & 1.57424 & \\
\hline & & & 734) & & & & & $(0.01288)$ & 50) \\
\hline \multirow[t]{2}{*}{50} & & & & & & & & & \\
\hline & & & & & & & & & \\
\hline \multirow{2}{*}{100} & 1.05975 & & & & & & & 068 & \\
\hline & $(0.00605)$ & $(0.00780)$ & $.00550)$ & $(0.00$ & $00997)$ & & $(0.0$ & $(0.00978)$ & \\
\hline \multirow{2}{*}{150} & & & & & & & & 2772 & \\
\hline & & & & & & & & $778)$ & \\
\hline \multirow{2}{*}{200} & 1.04619 & & & & & & & & \\
\hline & $(0.00370)$ & & & & & & & $(0.00608)$ & \\
\hline \multirow{2}{*}{20} & 2.52673 & & & & & & & 42 & \\
\hline & & & & & & & & & \\
\hline \multirow{2}{*}{50} & & & & & & & & & \\
\hline & & & & & & & & & \\
\hline \multirow{2}{*}{100} & & & & & & & & 344 & \\
\hline & $(0.0$ & & & & & & & 150) & 85 \\
\hline \multirow{2}{*}{150} & 2.0 & & & & & & & 0803 & \\
\hline & & & & & & & & & \\
\hline \multirow{2}{*}{200} & & & & & & & & 3.59792 & \\
\hline & & & & & & & & $(0.00714)$ & $26)$ \\
\hline \multirow{2}{*}{20} & & & & & & & & 3.14641 & \\
\hline & & & & & & & & & 47) \\
\hline \multirow{2}{*}{50} & 2.13724 & 3.51508 & 0.50906 & 2.28442 & 3.49254 & 0.51598 & 2.14991 & 3.30175 & 0.52290 \\
\hline & & & $(0.01050)$ & (0.018 & & & $(0.00$ & $(0.01404)$ & $916)$ \\
\hline \multirow{2}{*}{100} & 2.12656 & & & & & & & 3.41910 & \\
\hline & & & & & & & & $(0.01202)$ & 785) \\
\hline \multirow{2}{*}{150} & 2.06631 & 3.67627 & 0.51391 & 2.20860 & 3.65269 & 0.52090 & 2.07856 & 3.45315 & 0.52789 \\
\hline & & & & & & & & $(0.00957)$ & $(0.00625)$ \\
\hline 200 & & & 0.51813 & & & & & 3.54163 & 0.53222 \\
\hline & & & & & & & & $(0.00747)$ & 0.00488 \\
\hline
\end{tabular}




\section{Real life example}

This section covers the analysis of real life data set regarding the breaking strengths of 64 single carbon fibers of length 10, presented by [21]. The idea has been to see whether the results and properties of the Bayes estimators, explored by simulation study, are applicable to a real life situation. We have taken $n=64, r=7$ and $s=58$ in order to have censoring rate close to $20 \%$ (that has been used in simulation study). The results of the analysis have been reported in the following tables. The amounts of posterior risks associated with each estimate have been presented in the parenthesis in the tables.

Table 5 contain the Bayes estimates and posterior risks for the mixture distribution using real life data. In case of point estimation, the performance of improved informative priors is better than non-informative prior. The minimum amounts of posterior risks have been observed under the assumption of improved inverse levy prior using SLLF. Therefore, the findings from the analysis of real life data are in accordance with the simulation study.

Table 5. Bayes estimates and posterior risks under real life data.

\begin{tabular}{|c|c|c|c|c|c|c|c|c|c|}
\hline \multirow{3}{*}{ LF } & \multicolumn{9}{|c|}{$\pi=0.45$} \\
\hline & $\hat{\theta}_{1}$ & $\hat{\theta}_{2}$ & $\hat{\pi}$ & $\hat{\theta}_{1}$ & $\hat{\theta}_{2}$ & $\hat{\pi}$ & $\hat{\theta}_{1}$ & $\hat{\theta}_{2}$ & $\hat{\pi}$ \\
\hline & \multicolumn{3}{|c|}{ Non-informative Prior } & \multicolumn{3}{|c|}{ Improved Gamma Prior } & \multicolumn{3}{|c|}{ Prior } \\
\hline SLLF & $\begin{array}{c}254.532 \\
(0.51382)\end{array}$ & $\begin{array}{c}280.913 \\
(0.27110)\end{array}$ & $\begin{array}{c}0.390 \\
(0.20301)\end{array}$ & $\begin{array}{c}256.355 \\
(0.46501)\end{array}$ & $\begin{array}{c}280.383 \\
(0.24650)\end{array}$ & $\mid \begin{array}{c}0.392 \\
(0.18934)\end{array}$ & $\begin{array}{l}259.469 \\
(0.41666)\end{array}$ & $\begin{array}{c}283.789 \\
(0.22110)\end{array}$ & $\begin{array}{c}0.393 \\
(0.16351)\end{array}$ \\
\hline \multirow[t]{2}{*}{ SELF } & $\begin{array}{c}260.936 \\
(0.64342)\end{array}$ & $\begin{array}{c}268.699 \\
(0.33944)\end{array}$ & $\begin{array}{c}0.383 \\
(0.25420)\end{array}$ & $\begin{array}{l}262.806 \\
(0.58222)\end{array}$ & $\begin{array}{c}268.192 \\
(0.30864)\end{array}$ & $\begin{array}{c}0.385 \\
(0.23707)\end{array}$ & $\begin{array}{c}265.998 \\
(0.52205)\end{array}$ & $\mid \begin{array}{c}271.449 \\
(0.27651)\end{array}$ & $\begin{array}{c}0.386 \\
(0.20480)\end{array}$ \\
\hline & \multicolumn{3}{|c|}{$\begin{array}{c}\text { Improved Inverse Levy } \\
\text { Prior }\end{array}$} & \multicolumn{3}{|c|}{$\begin{array}{c}\text { Improved Exponential } \\
\text { Prior }\end{array}$} & \multicolumn{3}{|c|}{$\begin{array}{c}\text { Improved Chi Square } \\
\text { Prier }\end{array}$} \\
\hline SLLF & $\begin{array}{l}255.690 \\
(0.37755)\end{array}$ & $\begin{array}{c}279.349 \\
(0.18838)\end{array}$ & $\begin{array}{c}0.411 \\
(0.15292)\end{array}$ & $\begin{array}{l}250.825 \\
(0.46529)\end{array}$ & $\begin{array}{l}276.518 \\
(0.28342)\end{array}$ & $\begin{array}{c}0.468 \\
(0.23256)\end{array}$ & $\begin{array}{c}252.622 \\
(0.42104) \\
\end{array}$ & $\begin{array}{c}275.997 \\
(0.21789) \\
\end{array}$ & $\begin{array}{c}0.470 \\
(0.21692)\end{array}$ \\
\hline \multirow[t]{3}{*}{ SELF } & $\begin{array}{l}262.124 \\
(0.49625)\end{array}$ & $\begin{array}{c}267.203 \\
(0.24759)\end{array}$ & $\begin{array}{c}0.404 \\
(0.20105)\end{array}$ & $\begin{array}{l}257.136 \\
(0.61170)\end{array}$ & $\begin{array}{l}264.495 \\
(0.37267)\end{array}$ & $\begin{array}{c}0.460 \\
(0.30581)\end{array}$ & $\begin{array}{c}258.978 \\
(0.55353) \\
\end{array}$ & $\begin{array}{c}263.997 \\
(0.28646)\end{array}$ & $\begin{array}{c}0.462 \\
(0.28517)\end{array}$ \\
\hline & \multicolumn{9}{|c|}{$\pi=0.55$} \\
\hline & \multicolumn{3}{|c|}{ Non-informative Prior } & \multicolumn{3}{|c|}{ Improved Gamma Prior } & \multicolumn{3}{|c|}{$\begin{array}{l}\text { Improved Jeffreys Gamma } \\
\text { Prior }\end{array}$} \\
\hline SLLF & $\begin{array}{l}251.106 \\
(0.48973)\end{array}$ & $\mid \begin{array}{c}277.192 \\
(0.38929)\end{array}$ & $\begin{array}{c}0.475 \\
(0.25093)\end{array}$ & $\begin{array}{l}252.905 \\
(0.44321)\end{array}$ & $\begin{array}{l}276.670 \\
(0.35396)\end{array}$ & $\begin{array}{c}0.478 \\
(0.23402)\end{array}$ & \begin{tabular}{|c|}
255.977 \\
$(0.39713)$
\end{tabular} & $\begin{array}{l}280.030 \\
(0.31749)\end{array}$ & $\begin{array}{c}0.479 \\
(0.20210)\end{array}$ \\
\hline \multirow[t]{2}{*}{ SELF } & $\begin{array}{c}257.424 \\
(0.61939)\end{array}$ & $\begin{array}{c}265.140 \\
(0.48742)\end{array}$ & $\begin{array}{c}0.467 \\
(0.31420)\end{array}$ & $\begin{array}{l}259.269 \\
(0.56048)\end{array}$ & $\begin{array}{c}264.639 \\
(0.44319)\end{array}$ & $\begin{array}{c}0.469 \\
(0.29302)\end{array}$ & $\begin{array}{c}262.418 \\
(0.50255)\end{array}$ & $\begin{array}{c}267.854 \\
(0.39705)\end{array}$ & $\begin{array}{c}0.471 \\
(0.25313)\end{array}$ \\
\hline & \multicolumn{3}{|c|}{$\begin{array}{c}\text { Improved Inverse Levy } \\
\text { Prior }\end{array}$} & \multicolumn{3}{|c|}{$\begin{array}{c}\text { Improved Exponential } \\
\text { prior }\end{array}$} & \multicolumn{3}{|c|}{$\begin{array}{c}\text { Improved Chi Square } \\
\text { Prior } \\
\end{array}$} \\
\hline SLLF & \begin{tabular}{|l|}
252.249 \\
$(0.35985)$
\end{tabular} & $\begin{array}{l}275.649 \\
(0.27050)\end{array}$ & $\begin{array}{c}0.501 \\
(0.18900)\end{array}$ & $\begin{array}{c}247.449 \\
(0.44348)\end{array}$ & $\begin{array}{l}272.856 \\
(0.40698)\end{array}$ & $\begin{array}{c}0.571 \\
(0.28745)\end{array}$ & \begin{tabular}{|c|}
249.222 \\
$(0.40130)$ \\
\end{tabular} & $\begin{array}{l}272.341 \\
(0.31289)\end{array}$ & $\begin{array}{c}0.573 \\
(0.26811)\end{array}$ \\
\hline SELF & $\begin{array}{c}258.596 \\
(0.45497)\end{array}$ & $\begin{array}{c}263.663 \\
(0.33860)\end{array}$ & $\begin{array}{c}0.492 \\
(0.23667)\end{array}$ & $\begin{array}{c}253.675 \\
(0.56082)\end{array}$ & $\begin{array}{c}260.991 \\
(0.50965)\end{array}$ & $\begin{array}{c}0.561 \\
(0.35998)\end{array}$ & $\begin{array}{c}255.493 \\
(0.50748)\end{array}$ & $\begin{array}{l}260.500 \\
(0.39175)\end{array}$ & $\begin{array}{c}0.563 \\
(0.33569)\end{array}$ \\
\hline
\end{tabular}

\section{Hazard rate for the mixture of Burr type VII distribution}

The hazard rate is a useful way of describing the distribution of time to event because it has a natural interpretation that relates to the aging of a population. The hazard function is the risk of failure in a small time interval, given survival at the beginning of the time interval. As a function of time, a hazard function may be increasing; meaning as time increases the rate for failure increases, for example, when a patient is untreated for a disease such as cancer or the medication do not work properly; may be decreasing, for example, as a person is recovering from severe trauma like a surgery, or may be constant, meaning the rate of failure is the same regardless of how much time has passed. The constant hazard rate is mostly unrealistic. The hazard rate for the mixture of Burr type VII distribution has been compared under a range of parametric values. 
Hazard rate function for mixture of Burr Type VII distribution is

$$
H(t)=\frac{\pi \theta_{1} 2^{-\theta_{1}} \operatorname{sech}^{2}(t)\{1+\tanh (t)\}^{\theta_{1}-1}+(1-\pi) \theta_{2} 2^{-\theta_{2}} \operatorname{sech}^{2}(t)\{1+\tanh (t)\}^{\theta_{2}-1}}{1-\left\{\pi 2^{-\theta_{1}}\{1+\tanh (t)\}^{\theta_{1}}+(1-\pi) 2^{-\theta_{2}}\{1+\tanh (t)\}^{\theta_{2}}\right\}} .
$$

The graphs for the hazard rate of the mixture model, for different parametric values and for the various ranges of the variable, are presented in Figures 3 to 6 . The abbreviations in the graphs are: $\mathrm{H}(\mathrm{t})$ : Hazard rate; PR1: $\theta_{1}=0.50, \theta_{2}=0.75 ;$ PR2: $\theta_{1}=50, \theta_{2}=75$; PR3: $\theta_{1}=0.50, \theta_{2}=75$; PR4: $\theta_{1}=50, \theta_{2}=0.75$; PR5: $\theta_{1}=100, \theta_{2}=120$; PR6: $\theta_{1}=100, \theta_{2}=0.75 ;$ PR7: $\theta_{1}=0.50, \theta_{2}=120$.

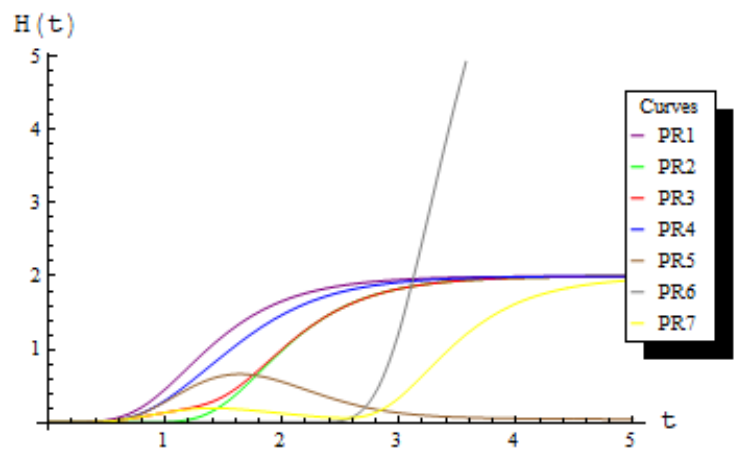

Figure 3. Graph of hazard rates for mixture of model using $\pi=0.25$.

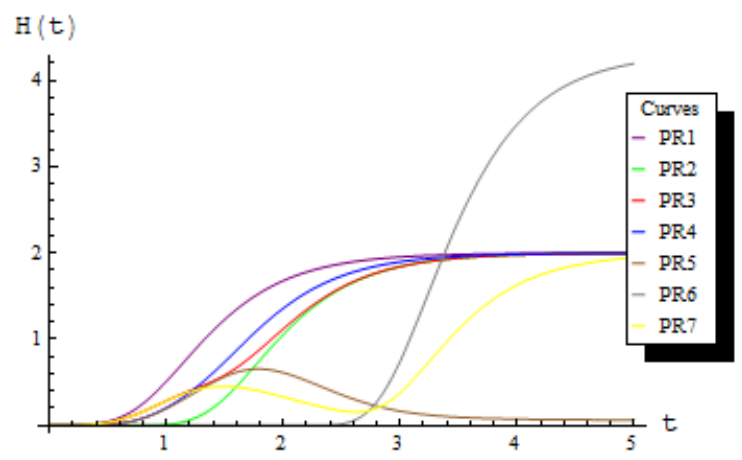

Figure 4. Graph of hazard rates for mixture of model using $\pi=0.75$.

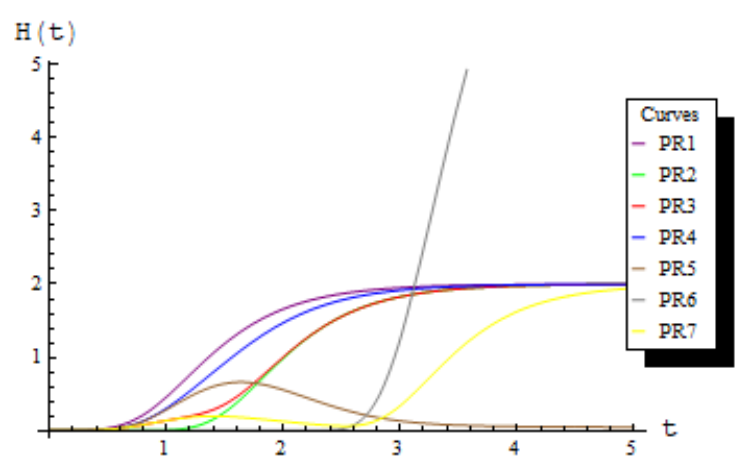

Figure 5. Graph of hazard rates for mixture of model using $\pi=0.50$. 


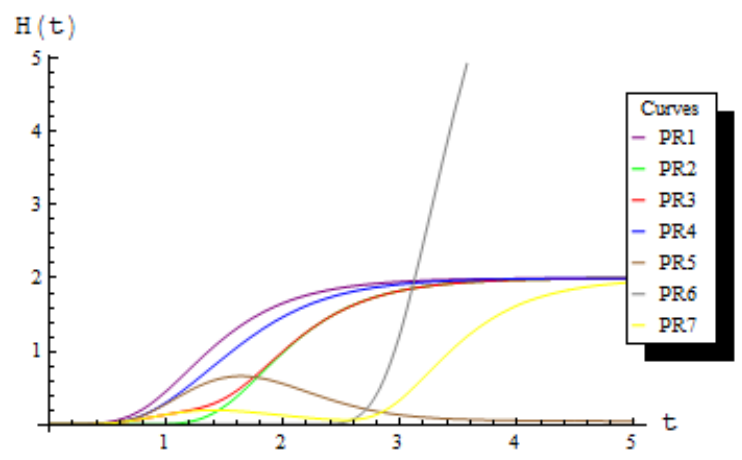

Figure 6. Graph of hazard rates for mixture of model using $\pi=0.90$.

The graphs suggest that the hazard rate for the mixture model tend to decrease for $t<2$ for closer choice of the values of the parameters. However, when the $\theta_{1}$ and $\theta_{2}$ differ significantly, the increase in the values of $t$ shows ebbs and flows in the curves of the hazard rate. Except PR5 and PR6, the curves of hazard rate functions tend to converge to each other for $t>4$.

\section{Conclusion}

The study has been carried out to introduce the methodology for Bayesian analysis of mixed lifetime models under doubly censored samples: with an application to Burr type VII distribution. A class of improved informative priors have been proposed and elicited for the analysis. A couple of loss functions have been assumed for the Bayes estimation. After a detailed analysis, it has been assessed that the improved inverse levy prior has provided the supreme results. Furthermore, the employment of the squared logarithmic loss function can produce the significantly better estimates for the parameters of the mixed Burr type VII distribution. The proposed estimators are consistent and capable of providing the stable results from moderate to large samples. The analysis of real life data further strengthened the findings of the simulation study. The study is useful for the analysts from different fields dealing with the analysis of the lifetime models when cases of failure are more than one and the duration time of the analysis is between two events.

\section{References}

[1] W.M. Afify, Classical estimation of mixed Rayleigh distribution in type I progressive censored, J. Stat. Theory Appl. 10 (4), 619-632, 2011.

[2] K.E. Ahmad, H.M. Moustafa and A.M. Abd-El-Rahman, Approximate Bayes estimation for mixtures of two Weibull distributions under type II censoring, J. Stat. Comput. Simul. 58 (3), 267-285, 1997.

[3] M. Aslam, An application of prior predictive distribution to elicit the prior density, J. Stat. Theory Appl. 2 (1), 183-197, 2003.

[4] L.D. Brown, Inadmissibility of the usual estimators of scale parameters in problems with unknown location and scale parameters, Ann. Math. Statist. 39 (1), 29-48, 1968.

[5] W.I. Burr, Cumulative frequency distribution, Ann. Math. Statist. 13 (2), 215-232, 1942.

[6] K.M. Chaloner, T. Church, T.A. Louis and J.P. Matts, Graphical elicitation of a prior distribution for a clinical trial, Statistician 42, 341-353, 1993.

[7] K.M. Chaloner and G.T. Duncan, Assessment of a Beta prior distribution: PM elicitation, Statistician 32, 174-180, 1983.

[8] E. Demidenko, Mixed Models: Theory and Applications, Wiley, 2004. 
[9] U. Erisoglu, M. Erisoglu and H. Erol, A mixture model of two different distributions approach to the analysis of heterogeneous survival data, World Acad Sci Eng Technol 78 (2), 41-45, 2011.

[10] N. Feroze and M. Aslam, On Bayesian analysis of Burr type VII distribution under different censoring schemes, Int. J. Qual. Reliab. Manag. 3, Doi:10.1155/2012/248146, 2012.

[11] N. Feroze and M. Aslam, Bayesian analysis of Burr type $X$ distribution under complete and censored samples, Int J Pure Appl Sci Technol 11 (2), 16-28, 2012.

[12] N. Feroze and M. Aslam, Bayesian analysis of Gumbel type II distribution under doubly censored samples using different loss functions, Casp. J. Appl. Sci. Res. 1 (10), 1-10, 2012.

[13] P.H. Garthwaite and J.M. Dickey, Elicitation of prior distributions for variableselection problems in regression, Ann. Statist. 20 (4), 1697-1719, 1992.

[14] C.F. Gauss, Least Squares Method for the Combinations of Observations, MalletBachelier, 1810.

[15] E.A. Gehan, A generalized two-sample Wilcoxon test for doubly censored data, Biometrika 52 (3/4), 650-653, 1965.

[16] S. Geisser, A Predictive Primer, Bayesian Analysis in Econometrics and Statistics, in: A. Zellner (ed.), Amsterdam: North-Holland, 1980.

[17] A. Gelman, J.B. Carlin, H.S. Stern and D.B. Rubin, Bayesian Data Analysis, 2nd ed., Chapman and Hall/CRC, 2004.

[18] A. Haq, New improved informative priors for variance of Normal distribution, Available at: http://interstat.statjournals.net/YEAR/2009/abstracts/0911003. php.

[19] S.A. Ismail and I.H. El-Khodary, Characterization of mixtures of exponential family distributions through conditional expectation, in: Annual Conference on Statistics and Computer Modeling in Human and Social Sciences, 13, 64-73, 2001.

[20] P.S. Laplace, Theorie Analytique Des Probabilities, Veuve Courcier, 1812.

[21] J.F. Lawless, Statistical Models time Data, 2nd ed., Wiley, 2003.

[22] A.M. Legendre, New Methods for the Determination of Orbits of Comets, Courcier, 1805.

[23] B.G. Lindsay, Mixture Models: Theory, Geometry and Applications, The Institute of Mathematical Statistical, Hayward, CA, 1995.

[24] G.J. Maclachlan and D. Peel, Finite Mixture Models, Wiley, 2000.

[25] M.Y. Majeed and M. Aslam, Bayesian analysis of the two component mixture of inverted exponential distribution under quadratic loss functions, Int. J. Phys. Sci. 7 (9), 1424-1434, 2012.

[26] C.E. Mcculloch and S.R. Searle, Generalized, Linear and Mixed Models, Wiley, 2001.

[27] W. Mendenhall and R.J. Hadar, Estimation of Parameters of Mixed Exponential Distributions from Censored Life Test Data, John Wiley and Sons, 1958.

[28] M.A.M. Mousa and Z.F. Jaheen, Statistical inference for the Burr model based on progressively censored data, Comput. Math. Appl. 43 (10-11), 1441-1449, 2002.

[29] J.A. Nachlas and A. Kumar, Reliability estimation using doubly censored field data, IEEE Trans. Rel. 42 (2), 268-278, 1993.

[30] M.T. Nair and E.S. Abdul, Finite mixture of exponential model and its applications to renewal and reliability theory, J. Stat. Theory Pract. 4 (3), 367-373, 2010.

[31] M.M. Nassar, Two properties of mixtures of exponential distributions, IEEE Trans. Rel. 37 (4), 383-385, 1988.

[32] M.M. Nassar and M.R. Mahmoud, On characterizations of a mixture of exponential distributions, IEEE Trans. Rel. 34 (5), 484-488, 1985.

[33] H. Panahi and S. Asadi, Analysis of the type-II hybrid censored Burr type XII distribution under linex loss function, Appl. Math. Sci. 5 (79), 3929-3942, 2011. 
[34] P.G. Peer, J.A. Van Dijck, J.H. Hendriks and A.L. Verbeek, Age dependent growth data of primary breast cancer, Cancer 71 (11), 3547-3551, 1993.

[35] M. Raqab and M. Madi, Bayesian prediction of the total time on test using doubly censored Rayleigh data, J. Stat. Comput. Simul. 72 (10), 781-789, 2002.

[36] M. Saleem and M. Aslam, Bayesian analysis of the two component mixture of the Rayleigh dist. With the uniform and the Jeffreys priors, J. Appl. Stat. Sci. 16 (4), 105-113, 2008.

[37] M. Saleem and M. Aslam, On prior selection for the mixture of Rayleigh distribution using predictive Intervals, Pak. J. Statist. 24 (1), 21-35, 2008.

[38] M. Saleem, M. Aslam and P. Economou, On the Bayesian analysis of the mixture of power function distribution using the complete and the censored sample, J. Appl. Stat. 37 (1), 25-40, 2010.

[39] M. Saleem and M. Irfan, On properties of the Bayes estimates of the Rayleigh mixture parameters: a simulation study, Pak. J. Statist. 26 (3), 547-555, 2010.

[40] Q. Shao, Notes on maximum likelihood estimation for the three-parameter Burr XII distribution, Comput. Stat. Data Anal. 45 (3), 675-687, 2004.

[41] Q. Shao, H. Wong and J. Xia, Models for extremes using the extended three parameter Burr XII system with application to flood frequency analysis, Hydrol. Sci. J. 49 (4), 685-702, 2004.

[42] G.O. Silva, E.M. Ortega, V.G. Cancho and M.L. Barreto, Log-Burr XII regression models with censored data, Comput. Statist. Data Anal. 52 (7), 3820-3842, 2008.

[43] A.A. Soliman, Reliability estimation in a generalized life model with application to the Burr-XII, IEEE Trans. Rel. 51 (3), 337-343, 2002.

[44] A.A. Soliman, Estimation of parameters of life from progressively censored data using Burr-xii model, IEEE Trans. Rel. 54 (1), 34-42, 2005.

[45] K.S. Sultan, M.A. Ismail and A.S. Al-Moisheer, Mixture of two inverse Weibull distributions: properties and estimation, Comput. Statist. Data Anal. 51 (11), 5377-5387, 2007.

[46] J.G. Surles and W.J. Padgett, Inference for reliability and stress-length for a scaled Burr type x distribution, Lifetime Data Anal. 7 (2), 187-202, 2001.

[47] D.M. Titterington, A.F.M. Smith and U.E. Makov, Statistical Analysis of Finite Mixture Distributions, Wiley, 1985.

[48] B.W. Turnbull, Non-Parametric estimation of a survivorship function with doubly censored data, J. Amer. Statist. Assoc. 69 (345), 169-173, 1974.

[49] A.S. Wahed, Bayesian inference using Burr model under asymmetric loss function: an application to carcinoma survival data, J. Statist. Res. 40 (1), 45-57, 2006.

[50] S.J. Wu, Y.J. Chen and C.T. Chang, Statistical inference based on progressively censored samples with random removals from the Burr type xii distribution, J. Stat. Comput. Simul. 77 (1), 19-27, 2007.

[51] J.W. Wu and H.Y. Yu, Statistical inference about the shape parameter of the Burr type xii distribution under the failure-censored sampling plan, Appl. Math. Comput. 163 (1), 443-482, 2005. 